\title{
SEARCH FOR GeV GAMMA-RAY BURSTS WITH THE ARGO-YBJ DETECTOR: SUMMARY OF EIGHT YEARS OF OBSERVATIONS
}

\author{
B. Bartoli ${ }^{1,2}$, P. Bernardini ${ }^{3,4}$, X. J. Bi ${ }^{5}$, P. Branchini $^{6}$, A. Budano $^{6}$, P. Camarri $^{7,8}$, Z. CAO $^{5}$, R. CARdarelli $^{8}$, \\ S. Catalanotti ${ }^{1,2}$, S. Z. Chen $^{5}$, T. L. Chen $^{9}$, P. Creti $^{4}$, S. W. Cui ${ }^{10}$, B. Z. Dai ${ }^{11}$, A. D' Amone ${ }^{3,4}$, Danzengluobu ${ }^{9}$, \\ I. De Mitri ${ }^{3,4}$, B. D’Ettorre Piazzoli ${ }^{1,2}$, T. Di Girolamo ${ }^{1,2,21}$, G. Di Sciascio ${ }^{8}$, C. F. Feng ${ }^{12}$ Zhaoyang Feng $^{5}$,

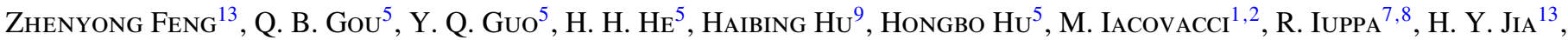 \\ LABACIREN $^{9}$, H. J. Li ${ }^{9}$, G. Liguori ${ }^{14,15}$, C. LiU ${ }^{5}$, J. LiU ${ }^{11}$, M. Y. LiU ${ }^{9}$, H. LU ${ }^{5}$, L. L. MA ${ }^{5}$, X. H. MA ${ }^{5}$, G. MANCARElla ${ }^{3,4}$, \\ S. M. Mari ${ }^{6,16}$, G. Marsella ${ }^{3,4}$, D. Martello ${ }^{3,4}$, S. Mastroianni ${ }^{2}$, P. Montini ${ }^{6,16}$, C. C. Ning ${ }^{9}$, M. Panareo ${ }^{3,4}$,

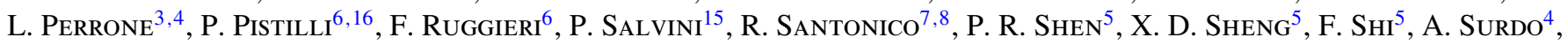 \\ Y. H. Tan ${ }^{5}$, P. Vallania ${ }^{17,18,21}$, S. Vernetto ${ }^{17,18}$, C. Vigorito ${ }^{18,19,21}$, H. Wang ${ }^{5}$, C. Y. Wu ${ }^{5}$, H. R. Wu ${ }^{5}$, L. Xue ${ }^{12}$,

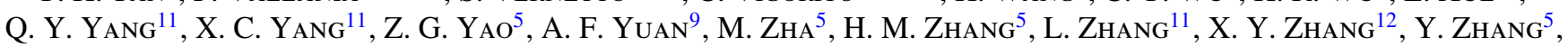

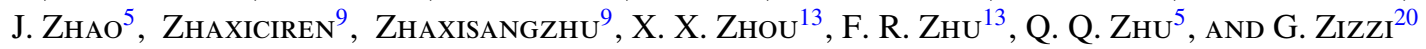 \\ (The ARGO-YBJ COLlaboration) \\ ${ }^{1}$ Dipartimento di Fisica dell'Università di Napoli “Federico II,” Complesso Universitario di Monte Sant'Angelo, \\ via Cinthia, I-80126 Napoli, Italy; digirola@na.infn.it \\ ${ }^{2}$ Istituto Nazionale di Fisica Nucleare, Sezione di Napoli, Complesso Universitario di Monte Sant'Angelo, via Cinthia, I-80126 Napoli, Italy \\ ${ }^{3}$ Dipartimento Matematica e Fisica "Ennio De Giorgi," Università del Salento, via per Arnesano, I-73100 Lecce, Italy \\ ${ }^{4}$ Istituto Nazionale di Fisica Nucleare, Sezione di Lecce, via per Arnesano, I-73100 Lecce, Italy \\ ${ }^{5}$ Key Laboratory of Particle Astrophysics, Institute of High Energy Physics, Chinese Academy of Sciences, P.O. Box 918, 100049 Beijing, Republic of China \\ ${ }^{6}$ Istituto Nazionale di Fisica Nucleare, Sezione di Roma Tre, via della Vasca Navale 84, I-00146 Roma, Italy \\ ${ }^{7}$ Dipartimento di Fisica dell'Università di Roma "Tor Vergata," via della Ricerca Scientifica 1, I-00133 Roma, Italy \\ ${ }^{8}$ Istituto Nazionale di Fisica Nucleare, Sezione di Roma Tor Vergata, via della Ricerca Scientifica 1, I-00133 Roma, Italy \\ ${ }^{9}$ Tibet University, 850000 Lhasa, Xizang, Republic of China \\ ${ }^{10}$ Hebei Normal University, Shijiazhuang 050016, Hebei, Republic of China \\ ${ }^{11}$ Yunnan University, 2 North Cuihu Rd., 650091 Kunming, Yunnan, Republic of China \\ ${ }^{12}$ Shandong University, 250100 Jinan, Shandong, Republic of China \\ ${ }^{13}$ Southwest Jiaotong University, 610031 Chengdu, Sichuan, Republic of China \\ ${ }^{14}$ Dipartimento di Fisica dell'Università di Pavia, via Bassi 6, I-27100 Pavia, Italy \\ 15 Istituto Nazionale di Fisica Nucleare, Sezione di Pavia, via Bassi 6, I-27100 Pavia, Italy \\ ${ }^{16}$ Dipartimento di Fisica dell'Università "Roma Tre," via della Vasca Navale 84, I-00146 Roma, Italy \\ ${ }^{17}$ Osservatorio Astrofisico di Torino dell’Istituto Nazionale di Astrofisica, via P. Giuria 1, I-10125 Torino, Italy; Piero.Vallania@to.infn.it \\ ${ }^{18}$ Istituto Nazionale di Fisica Nucleare, Sezione di Torino, via P. Giuria 1, I-10125 Torino, Italy; vigorito@to.infn.it \\ ${ }^{19}$ Dipartimento di Fisica dell'Università di Torino, via P. Giuria 1, I-10125 Torino, Italy \\ ${ }^{20}$ Istituto Nazionale di Fisica Nucleare-CNAF, Viale Berti-Pichat 6/2, I-40127 Bologna, Italy \\ Received 2014 May 26; accepted 2014 August 5; published 2014 September 25
}

\begin{abstract}
The search for gamma-ray burst (GRB) emission in the energy range of 1-100 GeV in coincidence with the satellite detection has been carried out using the Astrophysical Radiation with Ground-based Observatory at YangBaJing (ARGO-YBJ) experiment. The high-altitude location (4300 $\mathrm{m}$ a.s.l.), the large active surface $\left(\sim 6700 \mathrm{~m}^{2}\right.$ of Resistive Plate Chambers), the wide field of view ( $\sim 2 \mathrm{sr}$, limited only by the atmospheric absorption), and the high duty cycle $(>86 \%)$ make the ARGO-YBJ experiment particularly suitable to detect short and unexpected events like GRBs. With the scaler mode technique, i.e., counting all the particles hitting the detector with no measurement of the primary energy and arrival direction, the minimum threshold of $\sim 1 \mathrm{GeV}$ can be reached, overlapping the direct measurements carried out by satellites. During the experiment lifetime from 2004 December 17 to 2013 February 7 , a total of 206 GRBs occurring within the ARGO-YBJ field of view (zenith angle $\theta \leqslant 45^{\circ}$ ) have been analyzed. This is the largest sample of GRBs investigated with a ground-based detector. Two light curve models have been assumed and since in both cases no significant excess has been found, the corresponding fluence upper limits in the $1-100 \mathrm{GeV}$ energy region have been derived, with values as low as $10^{-5} \mathrm{erg} \mathrm{cm}^{-2}$. The analysis of a subset of 24 GRBs with known redshift has been used to constrain the fluence extrapolation to the GeV region together with possible cutoffs under different assumptions on the spectrum.
\end{abstract}

Key words: astroparticle physics - gamma-ray burst: general

Online-only material: color figures

\section{INTRODUCTION}

Gamma-ray bursts (GRBs) are among the most powerful sources in the sky, covering a very wide energy range from radio to multi-GeV $\gamma$-rays. Even though they are located at

\footnotetext{
$\overline{21}$ To whom any correspondence should be addressed.
}

cosmological distances (Costa et al. 1997), at higher energies they outshine all other sources, including the Sun, during their typical duration of a few seconds. GRBs occur at an average rate of a few per day, coming from the whole universe. Their highenergy spectrum shows different features, the most important being a peak in the $\mathrm{keV}-\mathrm{MeV}$ region. There are at least two classes of GRBs, classified in terms of burst duration. 
Short GRBs last up to $2 \mathrm{~s}$ and show a harder spectrum with a typical peak energy in the $v F_{v}$ spectrum at Earth at $\sim 490 \mathrm{keV}$ (Nava et al. 2011). Their origin is believed to be due to the merging of two compact objects like neutron stars or a neutron star and a black hole (Ruffert \& Janka 1999; Rosswog et al. 2003). Recent support for this model comes from the optical and near-infrared detection of a faint transient, known as "kilonova," in the days following the short GRB 130603B (Tanvir et al. 2013). Long GRBs have duration greater than $2 \mathrm{~s}$ with a softer spectrum and a typical $v F_{v}$ peak around $160 \mathrm{keV}$ (Nava et al. 2011). In this case, the origin is believed to be due to the core collapse of Type Ic supernovae, and indeed the coincidence of the two events has been observed in several cases (see, for example, Weiler et al. 2001; Stanek et al. 2003; Gal-Yam et al. 2004; Campana et al. 2006). Most of the GRB spectra can be described by the Band function (Band et al. 1993), composed of two smoothly joined power laws. This function fits quite successfully the convex shape and broad peak of the spectral energy distribution of the GRB prompt emission; however, being a phenomenological model, it does not take into account any physical explanation concerning either the acceleration processes or non-thermal radiative losses. Despite the bulk emission being concentrated in the $\mathrm{keV}-\mathrm{MeV}$ energy region, the EGRET (Kanbach et al. 1988) and more recently the Fermi (Meegan et al. 2009) and AGILE (Longo et al. 2012) satellites observed photons in the $\mathrm{MeV}-\mathrm{GeV}$ range.

At the time of writing this paper, the highest photon energy measured at Earth is $95 \mathrm{GeV}$, observed by the Large Area Telescope (LAT) instrument on the Fermi satellite from GRB 130427A (Ackermann et al. 2014). The highest intrinsic energy $(\sim 147 \mathrm{GeV})$ detected from a GRB comes from a $27.4 \mathrm{GeV} \gamma$-ray observed during GRB 080916C, which has a redshift of 4.35 . This $\gamma$-ray was previously missed by the Fermi-LAT event analysis and was recently recovered using an improved data analysis (Atwood et al. 2013). Previously, the maximum observed photon energy was $33.4 \mathrm{GeV}$ from GRB 090902B ( $~ 94 \mathrm{GeV}$ when corrected for its redshift $z=1.822$ ). Until 2014 May, after almost six years of operation, Fermi-LAT detected photons above $10 \mathrm{GeV}$ from one short (GRB 090510) and eight long GRBs (Abdo et al. 2009a, 2009b; Ackermann et al. 2010, 2011, 2013, 2014; Kocevski et al. 2013; Vianello et al. 2013). Some of these GRBs (namely, GRB 08916C, GRB 090510, GRB 090902B, GRB 090926A, and GRB 130427A) cannot be well described at $\mathrm{GeV}$ energies with an extrapolation of the Band function seen at $\mathrm{keV}-\mathrm{MeV}$ energies, but require a much harder energy spectrum starting from $\sim 100 \mathrm{MeV}$ with a photon index $\alpha \sim-2$.

Another feature that characterizes the $\mathrm{GeV}$ emission is the light curve, with its onset delayed with respect to the $\mathrm{keV}-\mathrm{MeV}$ range and a longer duration, appearing as a very high energy afterglow. The current models include emission in both internal (Guetta \& Granot 2003; Finke et al. 2008) and external (Kumar \& Barniol Duran 2010; Ghisellini et al. 2010; Ghirlanda et al. 2010) shock scenarios, with $\gamma$-rays produced by leptonic or hadronic processes via inverse Compton scattering or neutral pion decay. The emission is believed to happen in highly relativistic narrow jets pointing toward the Earth. The study of the $\mathrm{GeV}$ energy region could be of great help in discriminating between different models. As an example, the delayed onset of the high-energy emission seen in most LAT-detected GRBs, if intrinsic, should favor the production from external shocks in the early GRB afterglow (Fan et al. 2008) instead of the reverse shock formed when the GRB ejecta encounter the interstellar medium (Wang et al. 2005).

GRBs have been detected through the whole universe, from the local one to redshift $z=8.2$, corresponding to $\sim 95 \%$ of the age of the universe. Unfortunately, the energy resolution of the instruments on board Fermi prevents the detection of clear spectral lines while their large angular uncertainty hampers the optical identification and follow-up. For these reasons, only the GRBs seen in the $\mathrm{keV}-\mathrm{MeV}$ region with arcmin resolution (as with Swift-BAT) have a measured redshift. In this same energy region, the spectral index is usually measured, but when the detected signal is weak, the time-averaged spectrum is poorly constrained. The absorption in the extragalactic background light (EBL) greatly reduces the high-energy photon flux from extragalactic sources. The detection of $>10 \mathrm{GeV}$ photons from high redshift sources can be used to constrain the EBL amount from regions where it is highly uncertain. Finally, the spectral slope in the $\mathrm{GeV}$ region could be of great help in discriminating between different GRB models. In particular, the detection of a cutoff energy could be indicative of $e$-pair production at source, allowing the measurement of the Lorentz boost factor of the jet (Ackermann et al. 2011). On the other hand, the spectral cutoff may be due to attenuation by the EBL, thus depending on the source redshift. GRBs at different distances could be used to disentangle these two effects.

At present, all the experimental data in the $\mathrm{MeV}-\mathrm{GeV}$ range have been obtained only from satellite detectors, which hardly cover the energy region above $1 \mathrm{GeV}$ due to their limited size and the fast decrease of the source energy spectra. Groundbased experiments can easily reach much larger effective areas exploiting two different techniques, which correspond to two different types of detectors: Extensive Air Shower (EAS) arrays and Imaging Atmospheric Cherenkov Telescopes (IACTs). Concerning the latter, the huge telescope recently installed at the HESS site or the planned Cherenkov Telescope Array (CTA) observatory can allow the detection of $\gamma$-rays with energy as low as 20-30 GeV (Becherini et al. 2012; Bernlöhr et al. 2013), even if only at moderate zenith angles. However, IACTs can operate only during nights with good weather conditions and no or limited moonlight, leading to a duty cycle of $10 \%-15 \%$. Another disadvantage is given by the limited full field of view, about $5^{\circ}$, which requires a fast slew after an external alert in order to observe a GRB, but as pointed out by Gilmore et al. (2013), the MAGIC experience shows that most observations started after considerably longer times despite the instrument rapid slew capabilities, with only a minority occurring with total delay times of $<100 \mathrm{~s}$, preventing the detection of short GRBs and the study of the very prompt phase of long GRBs. Due to the limited field of view, the prompt GRB location area must be quite small in order to be contained in it, but this is not the case for most of the GRBs detected by the Fermi-GBM. Until now, all the major Cherenkov telescope arrays (MAGIC, HESS, VERITAS) have attempted to detect a GRB with a follow up, but no robust positive result has been obtained and even with the new generation CTA, only $\lesssim 1$ detection per year is expected (Gilmore et al. 2013).

On the contrary, EAS arrays have a large field of view ( $\sim 2 \mathrm{sr})$ and a very high duty cycle (in principle, 100\%); however, the requirement of a sufficient number of secondary particles in order to reconstruct the shower arrival direction and primary energy leads to an energy threshold of at least $\sim 100 \mathrm{GeV}$. A possible technique to reduce the energy threshold of EAS detectors is working in scaler mode (Vernetto 2000) instead of 
shower mode, that is, recording the counting rates of the detector in search for an increase in coincidence with a burst detected by a different experiment. Even if this technique does not allow the reconstruction of the arrival direction and thus an independent search, it benefits from the large effective area and field of view and from the very low dead time with an energy threshold typically around $1 \mathrm{GeV}$, thus overlapping the highest energies investigated by satellites experiments. The resulting sensitivity is limited, but for GRBs observed at low zenith angles it is comparable to the highest fluxes measured by satellites.

The Astrophysical Radiation with Ground-based Observatory at YangBaJing (ARGO-YBJ) detector has operated in scaler mode from 2004 December 17 to 2013 February 7. In this period, a total of 206 GRBs (selected from the GCN Circulars Archive, ${ }^{22}$ the Second Fermi GBM Gamma-Ray Burst Catalog (von Kienlin et al. 2014; Gruber et al. 2014) and the Fermi GBM Burst Catalog Web site ${ }^{23}$ ) in the field of view of the detector were investigated searching for an increase in the detector counting rates. No significant excess has been found and corresponding upper limits to the fluence and energy cutoff under different assumptions on the spectrum are presented and discussed in this paper. A detailed description of the scaler mode technique, including the effective area calculation for gamma-rays and protons, the comparison between measured and simulated counting rates, the long-term counting rate behavior, and the detector stability over short and long time periods can be found in Aielli et al. (2008), while the analysis procedure is described in Aielli et al. (2009a) together with the results on the first sample of GRBs analyzed. The GRB search can be done both in shower and scaler mode; here only the results obtained with the latter are presented and discussed. Shower mode results on a reduced sample of GRBs are given in Aielli et al. (2009b).

\section{THE DETECTOR}

ARGO-YBJ (Aielli et al. 2012) is an EAS detector located at an altitude of $4300 \mathrm{~m}$ a.s.l. (atmospheric depth $606 \mathrm{~g} \mathrm{~cm}^{-2}$ ) at the YangBaJing Cosmic Ray Laboratory $(30.11 \mathrm{~N}, 90.53 \mathrm{E})$ in Tibet, P. R. China. It is mainly devoted to $\gamma$-ray astronomy (Aielli et al. 2010; Bartoli et al. 2011, 2012a, 2012b, 2012c, 2013a, 2013b) and cosmic ray physics (Aielli et al. 2009c, 2011; Bartoli et al. 2012d, 2012e, 2013c). The detector is made of a single layer of Resistive Plate Chambers (RPCs), operated in streamer mode and grouped into 153 units named "clusters" of size $5.7 \times 7.6 \mathrm{~m}^{2}$ (Aielli et al. 2006). Each cluster is made by 12 RPCs $\left(1.23 \times 2.85 \mathrm{~m}^{2}\right)$ and each RPC is read out by 10 pads $\left(55.6 \times 61.8 \mathrm{~cm}^{2}\right)$, representing the space-time pixels of the detector. The clusters are disposed in a central full-coverage carpet (130 clusters on an area $74 \times 78 \mathrm{~m}^{2}$ with $\sim 92 \%$ of active surface) surrounded by a partially instrumented $(\sim 20 \%)$ area up to $100 \times 110 \mathrm{~m}^{2}$, which increases the effective area and improves the reconstruction of the core location in shower mode.

In scaler mode, the total counts are measured every $0.5 \mathrm{~s}$ : for each cluster the signal coming from its 120 pads is added up and put in coincidence in a narrow time window (150 ns), giving the counting rates for $\geqslant 1, \geqslant 2, \geqslant 3$, and $\geqslant 4$ pads, which are read by four independent scaler channels. These counting rates are referred in the following as $C_{\geqslant 1}, C_{\geqslant 2}, C_{\geqslant 3}$, and $C_{\geqslant 4}$, respectively, and the corresponding rates are $\sim 40 \mathrm{kHz}, \sim 2 \mathrm{kHz}$, $\sim 300 \mathrm{~Hz}$, and $\sim 120 \mathrm{~Hz}$. Since for the GRB search in scaler

\footnotetext{
22 http://gcn.gsfc.nasa.gov/gcn3_archive.html

$23 \mathrm{http}: / /$ heasarc.gsfc.nasa.gov/W3Browse/fermi/fermigbrst.html
}

mode the authentication is only given by a satellite detection, the stability of the detector and the probability that it mimics a true signal are crucial and have to be deeply investigated.

The main sources of counting rate variations are the pressure, acting on the shower development in the atmosphere, and the ambient temperature, acting on the detector efficiency. The timescale of both variations is much larger than the typical GRB duration (seconds to minutes), so they can be neglected provided that the behavior of the single cluster counting rates is Poissonian. A secondary local effect is due to the radon contamination in the detector hall. Electrons and $\gamma$-rays from short-lived radon daughters (mainly ${ }_{82}^{214} \mathrm{~Pb},{ }_{83}^{214} \mathrm{Bi},{ }_{84}^{214} \mathrm{Po}$ ) produced in the radon decay chain are expected from $\beta$ decays and isotope de-excitations. It has been shown that they can influence the cluster counting rates at a level of a few percent of the reference value. Even in this case, the time variations are larger (hours) than the typical GRB duration and they can be neglected in the data processing (Bartoli et al. 2014; Giroletti et al. 2011).

A very rapid variation can be induced by nearby lightning. For this reason, two electric field monitors EFM-100 located at opposite sides of the experimental hall and a storm tracker $L D$ 250 (both devices by Boltek industries ${ }^{24}$ ) have been installed to check the electric field variations. Details of this study are widely discussed in Zhou et al. (2011).

\section{DATA SELECTION AND ANALYSIS}

The ARGO-YBJ detector was completed in the spring of 2007; however, thanks to its modularity, the data collection started already in 2004 November (corresponding to the launch of the Swift satellite), ending in 2013 February, when the detector was definitively switched off. In this period, a total of $223 \mathrm{GRBs}$ detected by satellite instruments, occurred inside the ARGOYBJ field of view (zenith angle $\theta \leqslant 45^{\circ}$, corresponding to $1.84 \mathrm{sr}$ ). The present analysis was carried out on 206 of them, since the other GRBs occurred during periods when the detector was inactive or not properly working. Unlike $\Delta t_{90}$, defined as the time during which $90 \%$ of the GRB keV-MeV photons are detected, the redshift and the spectrum in the same energy range are not always measured due to the difficulties introduced in Section 1. The spectra measured by satellites can be fitted with a simple power law, a smooth double power law (Band or smoothly broken power law, SBPL; Kaneko et al. 2006) or a cutoff power law (CPL). Figure 1(a) shows the $\Delta t_{90}$ distribution with the dashed area on the left indicating the short $(\leqslant 2 s)$ GRB population, while Figure 1(b) gives the distribution of the fluences measured by the satellites, all normalized to the energy interval $15-150 \mathrm{keV}$. For 103 GRBs of our sample, the simple power-law spectral index in the $\mathrm{keV}-\mathrm{MeV}$ region was measured by satellite detectors and the corresponding distribution (with a mean value $\langle\alpha\rangle=1.6$ ) is shown in Figure 1(c). For 24 of them, the redshift is also known and the corresponding distribution is shown in Figure 1(d), being $\langle z\rangle=2.1$, the mean value of this subset. The durations $\Delta t_{90}$, the fluences, and the spectral indices $\alpha$ of GRBs with known redshift are pointed out in Figures 1(a), (b), and (c), respectively, with a dashed area (colored red in the online version). For this subset, the mean value and width of the three distributions are compatible with those for the whole GRB sample. The detailed list of the 24 GRBs with known redshift is given in Table 1, while Table 2 reports the same information for the remaining $182 \mathrm{GRBs}$.

\footnotetext{
24 http://www.boltek.com/
} 

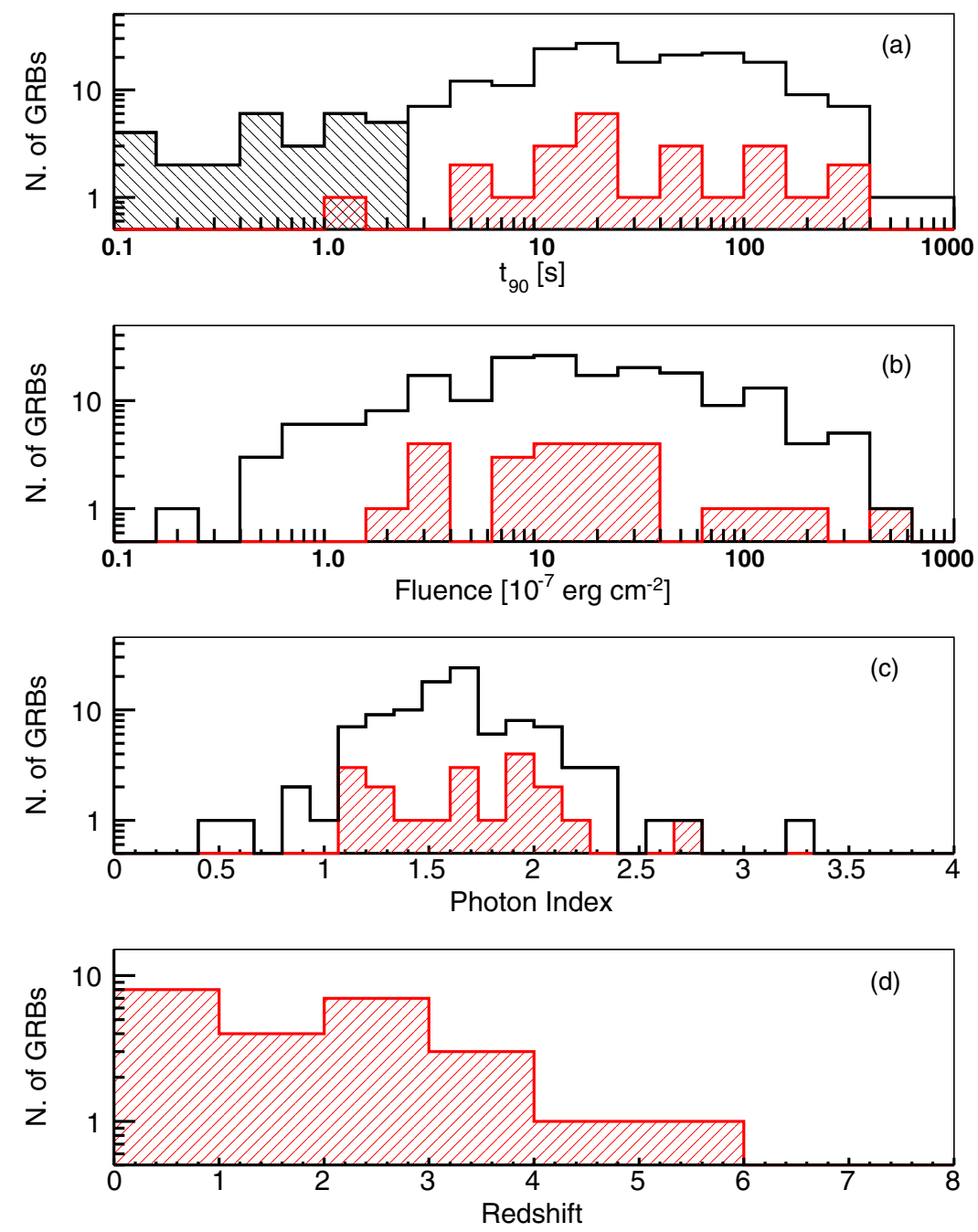

Figure 1. Details of the GRB sample analyzed in coincidence with ARGO-YBJ: (a) $\Delta t_{90}$ durations of the whole sample (solid line) and of the GRBs with known redshift (filled area); (b) fluences measured by satellites (all normalized to the energy range $15-150 \mathrm{keV}$ ) for the whole available sample (solid line) and for the events with known redshift (filled area); (c) photon index values in the $\mathrm{keV}-\mathrm{MeV}$ band for the whole available sample (solid line) and for the events with known redshift (filled area); and (d) redshift values distribution. The dashed area on the left in plot (a) indicates the short ( $\leqslant 2 \mathrm{~s})$ GRB population.

(A color version of this figure is available in the online journal.)

For each GRB, the following standard procedure has been adopted: check of the detector stability, cluster selection by means of quality cuts, and calculation of the significance of the coincident signal in the ARGO-YBJ detector. In order to extract the maximum information from the experimental data, two analyses have been implemented:

1. coincidence search for each GRB; and

2. cumulative search for stacked GRBs.

Details on quality cuts and detector stability are carefully discussed in Aielli et al. (2008), while the background evaluation and significance calculation, as well as the analysis technique itself, are described in Aielli et al. (2009a).

\subsection{Coincidence Search}

The counting rates of the clusters surviving the quality cuts (with an average efficiency over the whole data set of $\sim 87 \%$ ) are added up and the normalized fluctuation function

$$
f=(c-b) / \sigma, \sigma=\sqrt{b+b \frac{\Delta t_{90}[\mathrm{~s}]}{600}}
$$

is used to evaluate the significance of the excess observed in coincidence with the satellite detection, where $c$ is the total number of counts in the $\Delta t_{90}$ time window starting at $t_{0}$ (the trigger time) of the signal, both given by the satellite detector, and $b$ is the number of counts in a fixed time interval of $300 \mathrm{~s}$ before and after the signal, normalized to the $\Delta t_{90}$ time. This analysis can be done for the counting rates of all the multiplicities $\geqslant 1, \geqslant 2, \geqslant 3, \geqslant 4$ and $1,2,3$, where the counting rates $C_{i}$ are obtained from the measured counting rates $C_{\geqslant i}$ using the relation

$$
C_{i}=C_{\geqslant i}-C_{\geqslant i+1} \quad(i=1,2,3) .
$$

In the following, if not otherwise specified, all the results are for the counting rate $C_{1}$, which corresponds to the minimum primary energy in the ARGO-YBJ scaler mode. The detector stability over short time periods is discussed in Aielli et al. (2008), showing that the Poissonian behavior of the distribution of the normalized fluctuations $f$ is preserved provided that the total time window considered by the analysis (i.e., the signal interval $\Delta t_{90}$ plus the background interval $2 \times 300 \mathrm{~s}$ ) is less than 30 minutes. This condition is satisfied for all GRBs included in our data sample, therefore no long time corrections of the 
Table 1

GRBs with Measured Redshift Observed by ARGO-YBJ

\begin{tabular}{|c|c|c|c|c|c|c|c|c|c|c|c|c|c|}
\hline GRB & Satellite & $\begin{array}{l}\Delta t_{90} \\
(\mathrm{~s}) \\
(3)\end{array}$ & $\begin{array}{c}\Delta t_{90}^{\prime} \\
(\mathrm{s}) \\
(4)\end{array}$ & $\begin{array}{l}\theta \\
\left({ }^{\circ}\right) \\
(5)\end{array}$ & (6) & $\begin{array}{l}\sqrt{F} \\
(7)\end{array}$ & $\begin{array}{l}\text { Spectral Index } \\
\left(\alpha_{\text {sat }}\right) \\
(8)\end{array}$ & $\begin{array}{c}A_{\mathrm{det}} \\
\left(\mathrm{m}^{2}\right) \\
(9)\end{array}$ & (10) & (11) & $\begin{array}{c}\text { Fluence U.L. }{ }^{\mathrm{a}, \mathrm{b}} \\
\left(\mathrm{erg} \mathrm{cm}^{-2}\right)\left(f_{\text {sat }}\right) \\
(12)\end{array}$ & $\begin{array}{c}\text { Fluence U.L. } \mathrm{b}, \mathrm{c} \\
\left(\mathrm{erg} \mathrm{cm}^{-2}\right)\left(f_{2.5}\right) \\
(13)\end{array}$ & $\begin{array}{c}E_{\text {cut }} \text { U.L. }{ }^{\mathrm{d}} \\
(\mathrm{GeV}) \\
(14)\end{array}$ \\
\hline 050408 & HETE & 15 & 23.7 & 20.4 & 1.24 & 1.3 & CPL & 1560 & -2.12 & -2.90 & & $9.1 \times 10^{-5}$ & $\ldots$ \\
\hline 050802 & Swift & 19 & 31.4 & 22.5 & 1.71 & 1.2 & 1.54 & 1516 & 0.19 & -0.02 & $1.0 \times 10^{-4}$ & $2.1 \times 10^{-4}$ & 8 \\
\hline 060115 & Swift & 139.6 & 139.6 & 16.6 & 3.53 & 3.0 & CPL & 3985 & -1.02 & -1.02 & $\ldots$ & $7.6 \times 10^{-4}$ & $\ldots$ \\
\hline 060526 & Swift & 298 & 298.0 & 31.7 & 3.21 & 3.9 & 2.01 & 4029 & -1 & -1.00 & $1.8 \times 10^{-3}$ & $2.7 \times 10^{-3}$ & $\ldots$ \\
\hline 060714 & Swift & 115 & 115.0 & 42.8 & 2.71 & 4.9 & 1.93 & 5155 & -0.61 & -0.61 & $4.2 \times 10^{-3}$ & $6.9 \times 10^{-3}$ & $\ldots$ \\
\hline 060927 & Swift & 22.5 & 31.3 & 31.6 & 5.6 & 1.6 & CPL & 5242 & -0.14 & -0.47 & $\ldots$ & $5.1 \times 10^{-4}$ & $\ldots$ \\
\hline 061110A & Swift & 40.7 & 40.7 & 37.3 & 0.76 & 2.5 & 1.67 & 5545 & 0.01 & 0.01 & $6.7 \times 10^{-4}$ & $1.7 \times 10^{-3}$ & $\ldots$ \\
\hline $071112 \mathrm{C}$ & Swift & 15 & 22.0 & 18.4 & 0.82 & 1.5 & 1.09 & 5198 & 1.01 & 0.46 & $4.5 \times 10^{-5}$ & $1.4 \times 10^{-4}$ & $<2$ \\
\hline 081028A & Swift & 260 & 260.0 & 29.9 & 3.04 & 3.9 & 1.25 & 5805 & 0.37 & 0.37 & $1.1 \times 10^{-3}$ & $3.0 \times 10^{-3}$ & 4 \\
\hline 090424 & Swift & 48 & 57.2 & 33.1 & 0.54 & 2.0 & 1.19 & 5762 & 0.6 & 0.71 & $1.4 \times 10^{-4}$ & $4.5 \times 10^{-4}$ & $<2$ \\
\hline 090426 & Swift & 1.2 & $\ldots$ & 43.7 & 2.61 & 1.3 & 1.93 & 5805 & -1.08 & $\ldots$ & $8.0 \times 10^{-5}$ & $1.3 \times 10^{-4}$ & $\ldots$ \\
\hline 090529A & Swift & 100 & 107.1 & 19.9 & 2.63 & 2.2 & 2 & 5892 & -0.66 & -0.83 & $2.7 \times 10^{-4}$ & $4.0 \times 10^{-4}$ & $\ldots$ \\
\hline 090809A & Swift & 5.4 & 7.9 & 34.2 & 2.74 & 1.5 & 1.34 & 5718 & -1.12 & -0.72 & $3.5 \times 10^{-5}$ & $8.8 \times 10^{-5}$ & 4 \\
\hline 090902B & Fermi & 25 & 36.6 & 23.1 & 1.82 & 1.7 & 1.94 & 5762 & 1.09 & 0.55 & $1.4 \times 10^{-4}$ & $1.7 \times 10^{-4 \mathrm{e}}$ & $\ldots$ \\
\hline $100302 \mathrm{~A}$ & Swift & 17.9 & 21.4 & 44.6 & 4.81 & 1.8 & 1.72 & 5675 & 0.04 & 0.03 & $1.4 \times 10^{-3}$ & $1.8 \times 10^{-3}$ & $\ldots$ \\
\hline $100418 \mathrm{~A}$ & Swift & 7.0 & 10.9 & 18.7 & 0.62 & 1.5 & 2.16 & 5978 & -1.33 & -0.77 & $2.9 \times 10^{-5}$ & $4.2 \times 10^{-5}$ & $\ldots$ \\
\hline 110106B & Swift & 24.8 & 30.5 & 25.1 & 0.62 & 2.4 & 1.76 & 5675 & 2.25 & 2.09 & $2.5 \times 10^{-4}$ & $5.6 \times 10^{-4}$ & $\ldots$ \\
\hline $110128 \mathrm{~A}$ & Swift & 30.7 & 30.7 & 43.2 & 2.34 & 2.3 & 1.31 & 5675 & 2.39 & 2.39 & $1.1 \times 10^{-3}$ & $2.9 \times 10^{-3}$ & 16 \\
\hline $111211 \mathrm{~A}$ & $A G I L E$ & 15 & 18.4 & 20.3 & 0.48 & 1.8 & 2.77 & 5545 & 0.78 & 0.92 & $1.8 \times 10^{-4}$ & $1.4 \times 10^{-4}$ & $\ldots$ \\
\hline $120326 \mathrm{~A}$ & Swift & 69.6 & 69.6 & 40.9 & 1.8 & 2.7 & CPL & 6021 & -0.8 & -0.80 & $\ldots$ & $2.2 \times 10^{-3}$ & $\ldots$ \\
\hline $120716 \mathrm{~A}$ & $I P N$ & 230 & 230.0 & 35.7 & 2.49 & 3.9 & CPL & 5718 & -0.57 & -0.57 & $\ldots$ & $7.1 \times 10^{-3}$ & $\ldots$ \\
\hline $120722 \mathrm{~A}$ & Swift & 42.4 & 42.5 & 17.7 & 0.96 & 2.0 & 1.9 & 5848 & 1.23 & 1.17 & $1.8 \times 10^{-4}$ & $3.2 \times 10^{-4}$ & $\ldots$ \\
\hline $120907 \mathrm{~A}$ & Swift & 16.9 & 21.1 & 40.2 & 0.97 & 2.0 & 1.73 & 5892 & -1.55 & -1.49 & $2.4 \times 10^{-4}$ & $5.6 \times 10^{-4}$ & $\ldots$ \\
\hline 130131B & Swift & 4.3 & 6.2 & 27.2 & 2.54 & 1.6 & 1.15 & 5762 & 0.85 & 0.50 & $5.2 \times 10^{-5}$ & $1.4 \times 10^{-4}$ & $<2$ \\
\hline
\end{tabular}

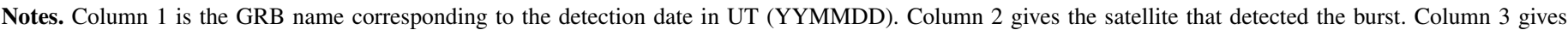

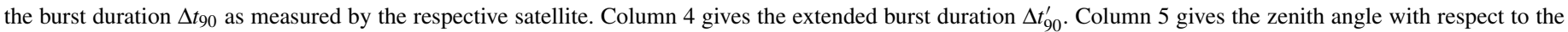

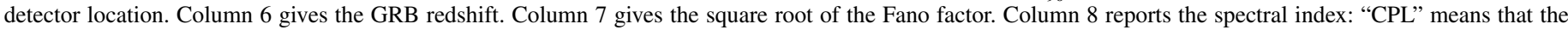

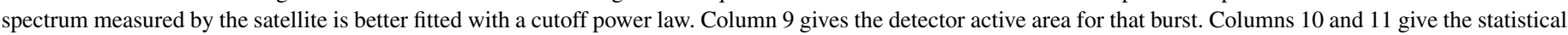

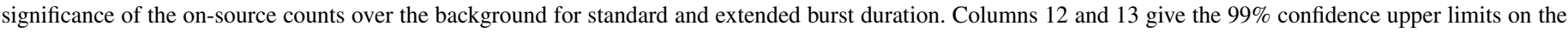
fluence between 1 and $100 \mathrm{GeV}$ for spectral index of Column 8 and fixed value -2.5 , respectively. Column 14 gives the cutoff upper limit, if any.

${ }^{a}$ Using the spectrum determined by satellites.

b $99 \%$ c.1.

c Assuming a differential spectral index 2.5.

d Derived from the $f_{\text {sat }}$ fluence U.L. (see the text).

e For high-energy emission extending with $\alpha_{\text {sat }}$ up to $30 \mathrm{GeV}$ only (see the text).

counting rates has been applied. Even if the distributions of the single cluster counter rates for integrated times up to half an hour are Poissonian, this is not true for the sum of different clusters, which shows larger fluctuations. This effect has been carefully analyzed and it was found to be due to the correlation between the counting rates of different clusters given by the air shower lateral distribution, i.e., counts in different clusters due to the same EAS are not independent. The resulting widening can be taken into account by introducing a Fano factor $F$ (Fano 1947):

$$
\sigma^{2}=F \sigma_{p}^{2}
$$

where $\sigma_{p}^{2}$ is the Poissonian variance equal to the mean value of the counting rate distribution and $\sigma^{2}$ is the measured variance. The Fano factor increases with the number of detector units used and the integration time (i.e., the GRB duration), while it decreases for a sparse detector layout and its effect is to reduce the sensitivity by a factor of $\sqrt{F}$. For each GRB, the $\sqrt{F}$ is listed in Tables 1 and 2 and the mean value calculated over the whole data sample is $\langle\sqrt{F}\rangle=2.22$. In order to take this effect into account and properly calculate the signal significance, we studied the local fluctuation of the normalized function $f$ (defined in Equation (1)) in an interval $\pm 12 h$ around the GRB trigger time and used Equation (17) of Li \& Ma (1983). Figure 2 (dark solid line) shows the distribution of the resulting significances for all the 206 GRBs. No significant excess is measured, the largest being $3.52 \sigma$ for GRB 080727C, with a post-trial chance probability of $4.5 \times 10^{-2}$. Since the long GRBs typically show a softer spectrum with a lower Band peak energy, the same distribution, but only for the 27 short GRBs, is shown in Figure 2 (dark dashed area, colored red in the online version). Even in this case, no significant excess is measured, with the most significant event being GRB 051114 with $3.37 \sigma$ and a post-trial chance probability of $1.0 \times 10^{-2}$. For this GRB, since we expect a harder energy spectrum from short GRBs, we carried out the same analysis using the higher multiplicity channels $C_{2}, C_{3}$, and $C_{\geqslant 4}$, obtaining a significance of $1.16 \sigma$, $1.09 \sigma$, and $1.95 \sigma$, respectively.

Besides this search, a time window broader than $\Delta t_{90}$ has been considered to take into account the possible high-energy afterglow. Ghisellini et al. (2010) found that the flux of 8 among the 11 brightest bursts detected by Fermi-LAT above $100 \mathrm{MeV}$ (in the first 13 months of operation) decays as a power law with a typical slope $t^{-1.5}$. In this analysis, we assumed this trend in 
Table 2

GRBs with No Measured Redshift ( $z=2$ and $z=0.6$ are Assumed for Long and Short GRBs, Respectively) Observed by ARGO-YBJ

\begin{tabular}{|c|c|c|c|c|c|c|c|c|c|c|c|c|}
\hline$\overline{\text { GRB }}$ & $\begin{array}{c}\text { Satellite } \\
\text { (2) }\end{array}$ & $\begin{array}{c}\Delta t_{90} \\
(\mathrm{~s}) \\
(3)\end{array}$ & $\begin{array}{c}\Delta t_{90}^{\prime} \\
(\mathrm{s}) \\
(4)\end{array}$ & $\begin{array}{c}\theta \\
\left({ }^{\circ}\right) \\
(5)\end{array}$ & $\sqrt{F}$ & $\begin{array}{c}\text { Spectral Index } \\
\left(\alpha_{\text {sat }}\right) \\
(7)\end{array}$ & $\begin{array}{c}A_{\mathrm{det}} \\
\left(\mathrm{m}^{2}\right) \\
(8)\end{array}$ & (9) & (10) & $\begin{array}{c}\text { Fluence U.L.a,b } \\
\left(\mathrm{erg} \mathrm{cm}^{-2}\right)\left(f_{\text {sat }}\right) \\
(11)\end{array}$ & $\begin{array}{l}\text { Fluence U.L. b,c } \\
\left(\mathrm{erg} \mathrm{cm}^{-2}\right)\left(f_{2.5}\right) \\
(12)\end{array}$ & $\begin{array}{c}E_{\text {cut U.L. }}{ }^{\mathrm{d}} \\
(\mathrm{GeV}) \\
(13)\end{array}$ \\
\hline 041228 & Swift & 55.4 & 68.4 & 28.1 & 1.5 & 1.6 & 563 & -0.01 & -0.27 & $5.8 \times 10^{-4}$ & $1.3 \times 10^{-3}$ & 52 \\
\hline 050509A & Swift & 11.4 & 16.9 & 34 & 1.3 & 2.11 & 1473 & 0.62 & 0.88 & $2.4 \times 10^{-4}$ & $3.5 \times 10^{-4}$ & $\ldots$ \\
\hline 050528 & Swift & 11.3 & 17.3 & 37.8 & 1.2 & 2.27 & 1473 & 0.71 & 0.04 & $1.0 \times 10^{-3}$ & $1.2 \times 10^{-3}$ & $\ldots$ \\
\hline 051105A & Swift & 0.1 & $\ldots$ & 28.5 & 1.2 & 1.22 & 3119 & 1.24 & $\ldots$ & $1.4 \times 10^{-5}$ & $4.5 \times 10^{-5}$ & 6 \\
\hline 051114 & Swift & 2 & 3.2 & 32.8 & 1.4 & 1.21 & 3032 & 3.37 & 3.27 & $5.0 \times 10^{-5}$ & $1.6 \times 10^{-4}$ & 5 \\
\hline 051227 & Swift & 114.6 & 114.6 & 22.8 & 2.9 & 1.45 & 2989 & 0.44 & 0.44 & $4.8 \times 10^{-4}$ & $1.1 \times 10^{-3}$ & 29 \\
\hline 060105 & Swift & 54.4 & 54.4 & 16.3 & 2.7 & 1.07 & 3119 & 1.77 & 1.77 & $3.3 \times 10^{-4}$ & $8.7 \times 10^{-4}$ & $<2$ \\
\hline 060111A & Swift & 13 & 19.6 & 10.8 & 1.5 & CPL & 3206 & 0.39 & 0.54 & & $1.1 \times 10^{-4}$ & $\ldots$ \\
\hline 060121 & HETE & 2 & 3.3 & 41.9 & 1.3 & 2.39 & 4159 & 0.6 & 0.58 & $2.2 \times 10^{-4}$ & $2.6 \times 10^{-4}$ & $\ldots$ \\
\hline 060421 & Swift & 12 & 19.3 & 39.3 & 1.3 & 1.55 & 3855 & -0.51 & -0.62 & $2.7 \times 10^{-4}$ & $6.2 \times 10^{-4}$ & 31 \\
\hline 060424 & Swift & 37.5 & 46.5 & 6.7 & 1.7 & 1.71 & 4072 & 0.12 & -0.11 & $9.5 \times 10^{-5}$ & $1.8 \times 10^{-4}$ & $\ldots$ \\
\hline 060427 & Swift & 64 & 76.6 & 32.6 & 1.8 & 1.87 & 4115 & -0.13 & -0.15 & $3.4 \times 10^{-4}$ & $6.0 \times 10^{-4}$ & $\ldots$ \\
\hline 060510A & Swift & 20.4 & 29.9 & 37.4 & 1.6 & 1.57 & 3899 & 2.42 & 2.31 & $9.2 \times 10^{-4}$ & $2.1 \times 10^{-3}$ & 21 \\
\hline 060717 & Swift & 3 & 4.9 & 7.4 & 1.5 & 1.7 & 5155 & 1.58 & 0.33 & $2.9 \times 10^{-5}$ & $5.5 \times 10^{-5}$ & $\ldots$ \\
\hline 060801 & Swift & 0.5 & $\ldots$ & 16.8 & 1.3 & 0.47 & 5415 & 0.81 & $\ldots$ & $7.4 \times 10^{-6}$ & $2.8 \times 10^{-5}$ & $<2$ \\
\hline 060805B & $I P N$ & 8 & 12.1 & 29.1 & 1.5 & 2.52 & 5285 & -0.45 & -0.17 & $1.3 \times 10^{-4}$ & $1.3 \times 10^{-4}$ & $\ldots$ \\
\hline 060807 & Swift & 54 & 54.0 & 12.4 & 2.6 & 1.58 & 5155 & 0.78 & 0.78 & $1.7 \times 10^{-4}$ & $3.4 \times 10^{-4}$ & 43 \\
\hline 061028 & Swift & 106 & 106.0 & 42.5 & 1.9 & 1.73 & 5458 & -3.33 & -3.33 & $4.7 \times 10^{-4}$ & $9.5 \times 10^{-4}$ & $\ldots$ \\
\hline 061122 & Integral & 18 & 18.0 & 33.5 & 3.9 & CPL & 5025 & 0.6 & 0.67 & $\ldots$ & $7.4 \times 10^{-4}$ & $\ldots$ \\
\hline 070201 & $I P N$ & 0.3 & $\ldots$ & 20.6 & 1.3 & CPL & 5242 & -1.21 & $\ldots$ & $\ldots$ & $1.1 \times 10^{-5}$ & $\ldots$ \\
\hline 070219 & Swift & 16.6 & 20.0 & 39.3 & 1.8 & 1.78 & 4982 & -0.71 & -0.76 & $4.2 \times 10^{-4}$ & $8.0 \times 10^{-4}$ & $\ldots$ \\
\hline 070306 & Swift & 209.5 & 209.5 & 19.9 & 3.4 & 1.66 & 2513 & -0.83 & -0.83 & $7.0 \times 10^{-4}$ & $1.3 \times 10^{-3}$ & $\ldots$ \\
\hline 070531 & Swift & 44.5 & 58.0 & 44.3 & 1.6 & 1.41 & 2816 & 0.59 & 0.65 & $9.1 \times 10^{-4}$ & $2.3 \times 10^{-3}$ & 24 \\
\hline 070615 & Integral & 30 & 37.1 & 37.6 & 1.7 & $\ldots$ & 5328 & 1.81 & 2.21 & $\ldots$ & $2.0 \times 10^{-3}$ & $\ldots$ \\
\hline 071013 & Swift & 26 & 32.1 & 13.3 & 1.9 & 1.6 & 4765 & -0.06 & -0.21 & $6.7 \times 10^{-5}$ & $1.4 \times 10^{-4}$ & $\ldots$ \\
\hline 071101 & Swift & 9 & 14.1 & 32.8 & 1.4 & 2.25 & 3596 & 1.01 & 0.53 & $2.0 \times 10^{-4}$ & $2.5 \times 10^{-4}$ & $\ldots$ \\
\hline 071104 & AGILE & 12 & 16.7 & 19.9 & 1.9 & $\ldots$ & 4029 & -0.07 & -0.24 & $\ldots$ & $1.5 \times 10^{-4}$ & $\ldots$ \\
\hline 071118 & Swift & 71 & 71.0 & 41.2 & 2.8 & 1.63 & 5025 & 0.54 & 0.54 & $1.8 \times 10^{-3}$ & $3.9 \times 10^{-3}$ & $\ldots$ \\
\hline 080328 & Swift & 90.6 & 90.6 & 37.2 & 2.7 & 1.52 & 6065 & -1.19 & -1.19 & $1.0 \times 10^{-3}$ & $2.4 \times 10^{-3}$ & 13 \\
\hline 080602 & Swift & 74 & 74.0 & 42 & 2.4 & 1.43 & 5762 & 1.24 & 1.24 & $1.5 \times 10^{-3}$ & $3.7 \times 10^{-3}$ & 18 \\
\hline 080613B & Swift & 105 & 105.0 & 39.2 & 2.6 & 1.39 & 5718 & 0.65 & 0.65 & $1.7 \times 10^{-3}$ & $4.3 \times 10^{-3}$ & 10 \\
\hline 080714B & Fermi & 5.4 & 8 & 24.4 & 1.5 & CPL & 5805 & -0.34 & -0.32 & $\ldots$ & $5.8 \times 10^{-5}$ & $\ldots$ \\
\hline 080727C & Swift & 79.7 & 79.7 & 34.5 & 2.1 & CPL & 5415 & 3.52 & 3.52 & $\ldots$ & $1.6 \times 10^{-3}$ & $\ldots$ \\
\hline 080730A & Fermi & 17.4 & 25.5 & 31.2 & 1.5 & 1.96 & 5545 & -0.26 & -0.87 & $1.2 \times 10^{-4}$ & $2.0 \times 10^{-4}$ & $\ldots$ \\
\hline 080822B & Swift & 64 & 65.8 & 40.3 & 2.4 & 2.54 & 5762 & -1.84 & -1.93 & $1.6 \times 10^{-3}$ & $1.5 \times 10^{-3}$ & $\ldots$ \\
\hline 080830 & Fermi & 45 & 45.0 & 37.1 & 2.1 & 1.69 & 5805 & -0.04 & -0.04 & $8.5 \times 10^{-4}$ & $1.8 \times 10^{-3}$ & $\ldots$ \\
\hline 080903 & Swift & 66 & 66.0 & 21.5 & 2.3 & CPL & 5588 & -1.33 & -1.33 & & $2.6 \times 10^{-4}$ & $\ldots$ \\
\hline 081025 & Swift & 23 & 32.6 & 30.5 & 1.6 & 1.12 & 5718 & -0.48 & -0.95 & $7.9 \times 10^{-5}$ & $2.3 \times 10^{-4}$ & $<2$ \\
\hline 081102B & Fermi & 2.2 & 3.3 & 27.8 & 1.4 & 1.07 & 5762 & 0.02 & -0.64 & $2.3 \times 10^{-5}$ & $6.8 \times 10^{-5}$ & $<2$ \\
\hline 081105 & $I P N$ & 10 & 15.1 & 36.7 & 1.5 & $\ldots$ & 5718 & -0.77 & -0.82 & & $4.7 \times 10^{-4}$ & $\ldots$ \\
\hline 081122 & Fermi & 26 & 30.7 & 8.3 & 1.8 & 2.24 & 4289 & -2.03 & -2.07 & $6.5 \times 10^{-5}$ & $8.1 \times 10^{-5}$ & $\ldots$ \\
\hline 081128 & Swift & 100 & 100.0 & 31.8 & 3.6 & CPL & 5242 & -0.63 & -0.63 & $\ldots$ & $1.1 \times 10^{-3}$ & $\ldots$ \\
\hline 081130B & Fermi & 12 & 14.7 & 28.6 & 2.3 & CPL & 5978 & -0.05 & 0.03 & $\ldots$ & $2.6 \times 10^{-4}$ & $\ldots$ \\
\hline $081215 \mathrm{~A}$ & Fermi & 7.7 & 10.3 & 35.9 & 1.9 & 2.20 & 5762 & -0.15 & 0.26 & $4.5 \times 10^{-4}$ & $6.0 \times 10^{-4}$ & $\ldots$ \\
\hline 090107A & Swift & 12.2 & 14.7 & 40.1 & 2.0 & 1.69 & 5762 & -1.12 & -1.59 & $3.0 \times 10^{-4}$ & $6.2 \times 10^{-4}$ & $\ldots$ \\
\hline 090118 & Swift & 16 & 21.1 & 13.4 & 1.9 & 1.35 & 5805 & -1.62 & -1.45 & $2.7 \times 10^{-5}$ & $6.3 \times 10^{-5}$ & 3 \\
\hline 090301 & Swift & 41.0 & 41.0 & 14.2 & 2.5 & CPL & 5805 & 0.73 & 0.73 & & $2.6 \times 10^{-4}$ & $\ldots$ \\
\hline 090301B & Fermi & 28 & 29.8 & 24.3 & 2.2 & 1.93 & 5892 & -2.2 & -2.15 & $7.8 \times 10^{-5}$ & $1.2 \times 10^{-4}$ & $\cdots$ \\
\hline 090306B & Swift & 20.4 & 20.4 & 38.5 & 2.3 & CPL & 5805 & -0.65 & -0.65 & $\ldots$ & $1.1 \times 10^{-3}$ & $\cdots$ \\
\hline 090320B & Fermi & 52 & 60.1 & 29 & 2.1 & CPL & 5892 & -0.25 & 0.04 & $\cdots$ & $4.9 \times 10^{-4}$ & $\cdots$ \\
\hline 090328B & Fermi & 0.32 & $\ldots$ & 15.5 & 1.3 & 2.48 & 5848 & 0.48 & $\ldots$ & $1.6 \times 10^{-5}$ & $1.7 \times 10^{-5}$ & \\
\hline 090403 & Fermi & 16 & 21.5 & 28.5 & 1.8 & $\ldots$ & 6021 & 0.65 & 1.16 & $\ldots$ & $2.9 \times 10^{-4}$ & $\cdots$ \\
\hline 090407 & Swift & 310 & 310.0 & 45 & 3.4 & 1.73 & 6021 & 1.53 & 1.53 & $6.7 \times 10^{-3}$ & $1.4 \times 10^{-2}$ & \\
\hline 090417B & Swift & 260 & 260.0 & 37.2 & 4.0 & 1.85 & 5978 & 0.64 & 0.64 & $6.2 \times 10^{-3}$ & $1.1 \times 10^{-2}$ & \\
\hline 090425 & Fermi & 72 & 92.0 & 44.6 & 1.9 & 2.03 & 5848 & 1.7 & 2.13 & $2.1 \times 10^{-3}$ & $3.3 \times 10^{-3}$ & . \\
\hline 090511 & Fermi & 14 & 17.9 & 39 & 1.7 & CPL & 5848 & 0.35 & 0.09 & & $8.8 \times 10^{-4}$ & $\ldots$ \\
\hline 090520A & Swift & 20 & 25.1 & 42.2 & 2.0 & 0.8 & 5892 & -0.65 & -0.57 & $2.6 \times 10^{-4}$ & $9.2 \times 10^{-4}$ & $\begin{array}{l}\cdots \\
<2\end{array}$ \\
\hline 090529C & Fermi & 10.4 & 15.6 & 22.1 & 1.4 & 2.1 & 5892 & 1.16 & 1.34 & $8.8 \times 10^{-5}$ & $1.2 \times 10^{-4}$ & $\ldots$ \\
\hline 090617 & Fermi & 0.45 & $\ldots$ & 16.1 & 1.4 & 2.00 & 5978 & 0.32 & $\ldots$ & $1.4 \times 10^{-5}$ & $2.4 \times 10^{-5}$ & $\cdots$ \\
\hline 090621B & Swift & 0.14 & $\ldots$ & 40.5 & 1.3 & 0.82 & 5935 & 0.5 & $\ldots$ & $2.4 \times 10^{-5}$ & $1.0 \times 10^{-4}$ & $<2$ \\
\hline 090704B & Fermi & 19.5 & 27.6 & 4.3 & 1.7 & 1.65 & 5848 & -0.66 & -0.37 & $3.7 \times 10^{-5}$ & $7.2 \times 10^{-5}$ & 28 \\
\hline 090712 & Swift & 145 & 145.0 & 10.7 & 3.7 & 1.33 & 5025 & -0.04 & -0.04 & $2.9 \times 10^{-4}$ & $6.9 \times 10^{-4}$ & 3 \\
\hline 090730A & Fermi & 9.1 & 14.6 & 4.4 & 1.4 & CPL & 5805 & 0.52 & -0.46 & & $5.9 \times 10^{-5}$ & $\ldots$ \\
\hline 090807A & Swift & 140.8 & 140.8 & 19.9 & 3.1 & 2.25 & 5935 & -0.76 & -0.76 & $5.1 \times 10^{-4}$ & $6.3 \times 10^{-4}$ & $\ldots$ \\
\hline 090807B & Fermi & 3 & 5.2 & 29.3 & 1.3 & 2.4 & 5978 & -1.14 & -2.69 & $5.1 \times 10^{-5}$ & $5.6 \times 10^{-5}$ & \\
\hline
\end{tabular}


Table 2

(Continued)

\begin{tabular}{|c|c|c|c|c|c|c|c|c|c|c|c|c|}
\hline GRB & $\begin{array}{l}\text { Satellite } \\
\text { (2) }\end{array}$ & $\begin{array}{l}\Delta t_{90} \\
(\mathrm{~s}) \\
(3)\end{array}$ & $\begin{array}{c}\Delta t_{90}^{\prime} \\
(\mathrm{s}) \\
(4)\end{array}$ & $\begin{array}{l}\theta \\
\left({ }^{\circ}\right) \\
(5)\end{array}$ & $\begin{array}{l}\sqrt{F} \\
(6)\end{array}$ & $\begin{array}{l}\text { Spectral Index } \\
\left(\alpha_{\text {sat }}\right) \\
(7)\end{array}$ & $\begin{array}{c}A_{\text {det }} \\
\left(\mathrm{m}^{2}\right) \\
(8)\end{array}$ & (9) & (10) & $\begin{array}{l}\text { Fluence U.L. }{ }^{\mathrm{a}, \mathrm{b}} \\
\left(\mathrm{erg} \mathrm{cm}^{-2}\right)\left(f_{\text {sat }}\right) \\
(11)\end{array}$ & $\begin{array}{l}\text { Fluence U.L. b,c } \\
\left(\mathrm{erg} \mathrm{cm}^{-2}\right)\left(f_{2.5}\right) \\
\text { (12) }\end{array}$ & $\begin{array}{c}E_{\text {cut }} \text { U.L. }^{\mathrm{d}} \\
(\mathrm{GeV}) \\
(13)\end{array}$ \\
\hline 090811A & Fermi & 14.8 & 21.7 & 23.1 & 1.7 & CPL & 5805 & -0.46 & -0.29 & $\ldots$ & $1.1 \times 10^{-4}$ & . \\
\hline 090814B & Integral & 50 & 50.5 & 31.1 & 2.2 & $\ldots$ & 5805 & -1.05 & -1.03 & $\ldots$ & $4.2 \times 10^{-4}$ & $\ldots$ \\
\hline 090817 & Integral & 220 & 220.0 & 14.6 & 2.9 & 2.2 & 5892 & -0.77 & -0.77 & $4.0 \times 10^{-4}$ & $5.2 \times 10^{-4}$ & $\ldots$ \\
\hline 090820A & Fermi & 30 & 41.2 & 17.1 & 1.7 & 2.61 & 5935 & 0.25 & 0.39 & $2.0 \times 10^{-4}$ & $1.9 \times 10^{-4}$ & $\ldots$ \\
\hline 090824A & Fermi & 59.9 & 69 & 30.8 & 1.7 & 2.01 & 5805 & 0.71 & 0.49 & $3.7 \times 10^{-4}$ & $5.8 \times 10^{-4}$ & $\ldots$ \\
\hline 090831A & Fermi & 53 & 67.9 & 35.8 & 2.8 & CPL & 4679 & 0.59 & 0.24 & $\ldots$ & $3.4 \times 10^{-3}$ & $\ldots$ \\
\hline 090904A & Swift & 122 & 164.8 & 21.9 & 1.9 & 2.01 & 5805 & 0.37 & 2.97 & $3.4 \times 10^{-4}$ & $5.1 \times 10^{-4}$ & $\ldots$ \\
\hline 090904C & Fermi & 38.4 & 46.6 & 33 & 1.9 & CPL & 5978 & -1.66 & -1.87 & $\ldots$ & $2.6 \times 10^{-4}$ & $\ldots$ \\
\hline 091106A & Fermi & 14.6 & 19.4 & 30.2 & 2 & CPL & 5762 & -0.17 & -0.32 & $\ldots$ & $2.5 \times 10^{-4}$ & $\ldots$ \\
\hline 091202 & Integral & 45 & 45.0 & 33.2 & 3.2 & $\ldots$ & 5415 & -0.64 & -0.64 & $\ldots$ & $6.5 \times 10^{-4}$ & $\ldots$ \\
\hline 091215A & Fermi & 4.4 & 6.1 & 25.4 & 1.5 & 1.65 & 5285 & -1.36 & -0.95 & $3.5 \times 10^{-5}$ & $7.4 \times 10^{-5}$ & 85 \\
\hline 091224A & Fermi & 0.8 & $\ldots$ & 16.8 & 1.3 & 1.21 & 5068 & -1.56 & $\ldots$ & $4.7 \times 10^{-6}$ & $1.4 \times 10^{-5}$ & $<2$ \\
\hline 091227A & Fermi & 21.9 & 23.6 & 27.9 & 2.1 & CPL & 5242 & 0.85 & 1.09 & $\ldots$ & $4.4 \times 10^{-4}$ & $\ldots$ \\
\hline $100111 \mathrm{~A}$ & Swift & 12.9 & 12.9 & 21.5 & 2.7 & 1.69 & 5458 & -1.03 & -1.03 & $7.5 \times 10^{-5}$ & $1.4 \times 10^{-4}$ & $\ldots$ \\
\hline $100115 \mathrm{~A}$ & Swift & 3 & 4.7 & 32.6 & 1.5 & $\ldots$ & 5588 & -0.29 & -0.14 & & $8.4 \times 10^{-5}$ & $\ldots$ \\
\hline $100122 \mathrm{~A}$ & Fermi & 6.6 & 9.3 & 33.1 & 1.6 & 2.31 & 5805 & 0.84 & 0.92 & $1.4 \times 10^{-4}$ & $1.7 \times 10^{-4}$ & $\ldots$ \\
\hline $100131 \mathrm{~A}$ & Fermi & 6.2 & 9.0 & 14 & 1.4 & 2.21 & 5588 & 1.01 & 1.16 & $4.8 \times 10^{-5}$ & $6.1 \times 10^{-5}$ & $\ldots$ \\
\hline 100206A & Swift & 0.12 & $\ldots$ & 26.8 & 1.2 & 0.63 & 4245 & 0.9 & $\ldots$ & $8.5 \times 10^{-6}$ & $3.4 \times 10^{-5}$ & $<2$ \\
\hline 100210A & Fermi & 29.2 & 29.2 & 24.9 & 2.4 & 1.71 & 5675 & 0.25 & 0.25 & $1.5 \times 10^{-4}$ & $2.7 \times 10^{-4}$ & $\ldots$ \\
\hline 100225B & Fermi & 32.0 & 38.4 & 22.1 & 1.9 & 1.51 & 5892 & -1.83 & -2.24 & $5.9 \times 10^{-5}$ & $1.2 \times 10^{-4}$ & 28 \\
\hline $100225 \mathrm{D}$ & Fermi & 4.5 & 7.1 & 8.4 & 1.5 & CPL & 5805 & -1.19 & -1.11 & $\ldots$ & $2.8 \times 10^{-5}$ & $\ldots$ \\
\hline $100424 \mathrm{~A}$ & Swift & 104 & 104.0 & 33.4 & 2.4 & 1.83 & 6021 & 0.41 & 0.41 & $5.3 \times 10^{-4}$ & $9.6 \times 10^{-4}$ & $\ldots$ \\
\hline $100503 \mathrm{~A}$ & Fermi & 129.5 & 129.5 & 26.4 & 4.5 & CPL & 6065 & 0.09 & 0.09 & $\ldots$ & $1.9 \times 10^{-3}$ & $\ldots$ \\
\hline 100513B & Fermi & 11.1 & 16.2 & 38.7 & 1.6 & CPL & 5502 & 1.16 & 1.17 & $\ldots$ & $9.0 \times 10^{-4}$ & $\ldots$ \\
\hline $100522 \mathrm{~A}$ & Swift & 35.3 & 46.0 & 27.7 & 1.7 & 1.89 & 4679 & 0.86 & 0.62 & $2.8 \times 10^{-4}$ & $4.9 \times 10^{-4}$ & $\ldots$ \\
\hline $100525 \mathrm{~A}$ & Fermi & 1.5 & 2.5 & 13.7 & 1.4 & CPL & 5892 & 0.65 & -0.16 & $\ldots$ & $2.2 \times 10^{-5}$ & $\ldots$ \\
\hline $100526 \mathrm{~A}$ & Swift & 102 & 102.7 & 9.5 & 2.5 & 1.83 & 5935 & -0.72 & -0.79 & $1.7 \times 10^{-4}$ & $2.8 \times 10^{-4}$ & $\ldots$ \\
\hline 100527A & Fermi & 184.6 & 297.4 & 33.3 & 2.2 & CPL & 5935 & 2.33 & 3.4 & $\ldots$ & $2.6 \times 10^{-3}$ & $\ldots$ \\
\hline $100530 \mathrm{~A}$ & Fermi & 3.3 & 5 & 39 & 1.4 & 1.66 & 6108 & 1.15 & 0.14 & $1.8 \times 10^{-4}$ & $3.9 \times 10^{-4}$ & $\ldots$ \\
\hline 100614B & Fermi & 172.3 & 197.9 & 43.2 & 2.4 & CPL & 5978 & -0.24 & -0.86 & $\ldots$ & $4.0 \times 10^{-3}$ & $\ldots$ \\
\hline $100621 \mathrm{C}$ & Fermi & 1.0 & $\ldots$ & 31.5 & 1.3 & $\ldots$ & 5762 & 0.71 & $\ldots$ & $\ldots$ & $4.3 \times 10^{-5}$ & $\ldots$ \\
\hline 100625B & Fermi & 29.2 & 36.4 & 15.4 & 1.7 & CPL & 5458 & 1.07 & 0.55 & $\ldots$ & $2.5 \times 10^{-4}$ & $\ldots$ \\
\hline 100706A & Fermi & 0.1 & $\ldots$ & 18.3 & 1.3 & 1.28 & 5675 & 0.39 & $\ldots$ & $5.6 \times 10^{-6}$ & $1.6 \times 10^{-5}$ & 4 \\
\hline $100713 \mathrm{~A}$ & Integral & 20 & 23.1 & 12.5 & 2.6 & $\ldots$ & 5848 & 0.43 & 0.37 & $\ldots$ & $1.7 \times 10^{-4}$ & $\ldots$ \\
\hline 100714B & Fermi & 5.6 & 8.7 & 39.9 & 1.5 & CPL & 5502 & -0.81 & -0.07 & $\ldots$ & $3.6 \times 10^{-4}$ & $\ldots$ \\
\hline $100718 \mathrm{~A}$ & Fermi & 38.7 & 47.6 & 16.7 & 2.1 & CPL & 5632 & -0.52 & -0.47 & $\ldots$ & $2.2 \times 10^{-4}$ & $\ldots$ \\
\hline $100728 \mathrm{~A}$ & Swift & 198.5 & 198.5 & 44.8 & 2.6 & 1.18 & 6021 & 0.49 & 0.49 & $2.0 \times 10^{-3}$ & $5.8 \times 10^{-3}$ & $<2$ \\
\hline $100902 \mathrm{~A}$ & Swift & 428.8 & 428.8 & 37 & 5.0 & 1.98 & 5415 & 0.41 & 0.41 & $1.2 \times 10^{-2}$ & $1.9 \times 10^{-2}$ & $\ldots$ \\
\hline 100929A & Fermi & 8.2 & 12.4 & 34.9 & 1.4 & 1.36 & 5892 & -0.38 & -0.68 & $4.8 \times 10^{-5}$ & $1.2 \times 10^{-4}$ & 9 \\
\hline 100929B & Fermi & 4.6 & 7.2 & 27.2 & 1.5 & 1.54 & 5848 & -0.68 & -0.61 & $4.0 \times 10^{-5}$ & $9.1 \times 10^{-5}$ & $\ldots$ \\
\hline $101003 \mathrm{~A}$ & Fermi & 10.0 & 15.3 & 30.8 & 1.5 & CPL & 5892 & -0.28 & -0.11 & $\ldots$ & $1.5 \times 10^{-4}$ & $\ldots$ \\
\hline 101008A & Swift & 104 & 104.0 & 25.6 & 2.4 & 1.59 & 5848 & 0.3 & 0.30 & $4.5 \times 10^{-4}$ & $9.8 \times 10^{-4}$ & $\ldots$ \\
\hline $101101 \mathrm{~A}$ & Fermi & 3.3 & 4.8 & 25.5 & 1.5 & 2.02 & 5935 & -0.02 & 0.57 & $5.5 \times 10^{-5}$ & $8.6 \times 10^{-5}$ & $\ldots$ \\
\hline $101107 \mathrm{~A}$ & Fermi & 375.8 & 375.8 & 25.8 & 3 & $\mathrm{CPL}$ & 5892 & 3 & 3 & $\ldots$ & $6.4 \times 10^{-3}$ & $\ldots$ \\
\hline 101112B & Fermi & 82.9 & 82.9 & 39.9 & 3.3 & CPL & 5805 & -0.95 & -0.95 & $\ldots$ & $3.0 \times 10^{-3}$ & $\ldots$ \\
\hline $101123 \mathrm{~A}$ & Fermi & 105 & 105.0 & 23.7 & 3.5 & 2.14 & 5978 & -0.79 & -0.79 & $3.1 \times 10^{-4}$ & $4.1 \times 10^{-4}$ & $\ldots$ \\
\hline $101202 \mathrm{~A}$ & Fermi & 18.4 & 26.3 & 38 & 1.5 & 1.62 & 5848 & -0.61 & -0.38 & $3.0 \times 10^{-4}$ & $6.6 \times 10^{-4}$ & $\ldots$ \\
\hline $101208 \mathrm{~A}$ & Fermi & 0.2 & $\ldots$ & 37.3 & 1.2 & CPL & 3899 & -0.42 & $\ldots$ & $\ldots$ & $8.9 \times 10^{-5}$ & $\ldots$ \\
\hline $101224 \mathrm{~A}$ & Swift & 0.2 & $\ldots$ & 22.6 & 1.3 & CPL & 5675 & -0.67 & $\ldots$ & $\ldots$ & $1.2 \times 10^{-5}$ & $\ldots$ \\
\hline $101231 \mathrm{~A}$ & Fermi & 23.6 & 23.6 & 24 & 2.5 & 2.44 & 5675 & -0.58 & -0.58 & $1.9 \times 10^{-4}$ & $1.9 \times 10^{-4}$ & $\ldots$ \\
\hline $110101 \mathrm{~A}$ & Fermi & 3.6 & 5 & 6.4 & 1.8 & 1.51 & 5848 & 0.21 & 0.23 & $2.1 \times 10^{-5}$ & $4.4 \times 10^{-5}$ & $\ldots$ \\
\hline $110106 \mathrm{~A}$ & Swift & 4.3 & 6.0 & 34.8 & 1.6 & 1.71 & 5588 & -0.28 & -0.71 & $3.8 \times 10^{-5}$ & $7.6 \times 10^{-5}$ & $\cdots$ \\
\hline $110206 \mathrm{~B}$ & Fermi & 12.3 & 17.8 & 43.4 & 1.5 & 1.55 & 5458 & -0.07 & 0.13 & $2.7 \times 10^{-4}$ & $6.2 \times 10^{-4}$ & $\begin{array}{l}\cdots \\
\cdots\end{array}$ \\
\hline $110210 \mathrm{~A}$ & Swift & 233 & 233.0 & 23 & 7.2 & 1.73 & 5762 & 1.15 & 1.15 & $1.9 \times 10^{-3}$ & $3.4 \times 10^{-3}$ & $\ldots$ \\
\hline $110220 \mathrm{~A}$ & Fermi & 33.0 & 33 & 31 & 2.2 & CPL & 5935 & 1.83 & 1.83 & $\ldots$ & $7.4 \times 10^{-4}$ & $\cdots$ \\
\hline $110226 \mathrm{~A}$ & Fermi & 14.1 & 17.7 & 37 & 1.8 & CPL & 5805 & -0.89 & -0.09 & $\ldots$ & $6.4 \times 10^{-4}$ & $\cdots$ \\
\hline $110312 \mathrm{~A}$ & Swift & 28.7 & 30.3 & 37.2 & 2.2 & 2.32 & 5805 & 0.3 & 0.21 & $1.3 \times 10^{-3}$ & $1.5 \times 10^{-3}$ & $\cdots$ \\
\hline $110315 \mathrm{~A}$ & Swift & 77 & 85.9 & 19.3 & 2.9 & 1.77 & 5112 & -2.26 & -2.58 & $1.6 \times 10^{-4}$ & $2.9 \times 10^{-4}$ & $\begin{array}{l}\cdots \\
\cdots\end{array}$ \\
\hline 110328B & Fermi & 40 & 40.0 & 20.8 & 2.6 & 3.31 & 6151 & 1.34 & 1.34 & $7.4 \times 10^{-4}$ & $4.4 \times 10^{-4}$ & $\begin{array}{l}\cdots \\
\cdots\end{array}$ \\
\hline $110401 \mathrm{~A}$ & Fermi & 2 & 3.5 & 15.2 & 1.3 & 2.36 & 5675 & -0.94 & -0.35 & $1.9 \times 10^{-5}$ & $2.2 \times 10^{-5}$ & $\begin{array}{l}\cdots \\
\cdots\end{array}$ \\
\hline $110406 \mathrm{~A}$ & Integral & 8 & 12.7 & 31.1 & 1.5 & 2.30 & 6108 & -0.1 & 0.07 & $1.1 \times 10^{-4}$ & $1.3 \times 10^{-4}$ & $\ldots$ \\
\hline $110414 \mathrm{~A}$ & Swift & 152.0 & 152.0 & 44.1 & 3.3 & 1.7 & 6021 & -0.92 & -0.92 & $2.0 \times 10^{-3}$ & $4.2 \times 10^{-3}$ & $\ldots$ \\
\hline 110517A & Fermi & 0.6 & $\ldots$ & 29.5 & 1.3 & 1.29 & 5198 & 2.55 & $\ldots$ & $2.1 \times 10^{-5}$ & $6.8 \times 10^{-5}$ & 13 \\
\hline $110605 \mathrm{~A}$ & Fermi & 82.7 & 82.7 & 33.8 & 3 & 2.20 & 6065 & -0.01 & -0.01 & $7.0 \times 10^{-4}$ & $9.3 \times 10^{-4}$ & $\ldots$ \\
\hline 110605B & Fermi & 1.5 & 2.6 & 39.9 & 1.4 & 1.5 & 5935 & 0.21 & 0.5 & $5.8 \times 10^{-5}$ & $1.7 \times 10^{-4}$ & 46 \\
\hline
\end{tabular}


Table 2

(Continued)

\begin{tabular}{|c|c|c|c|c|c|c|c|c|c|c|c|c|}
\hline GRB & $\begin{array}{l}\text { Satellite } \\
\text { (2) }\end{array}$ & $\begin{array}{c}\Delta t_{90} \\
(\mathrm{~s}) \\
(3)\end{array}$ & $\begin{array}{c}\Delta t_{90}^{\prime} \\
(\mathrm{s}) \\
(4)\end{array}$ & $\begin{array}{l}\theta \\
\left({ }^{\circ}\right) \\
(5)\end{array}$ & $\begin{array}{l}\sqrt{F} \\
(6)\end{array}$ & $\begin{array}{c}\text { Spectral Index } \\
\left(\alpha_{\text {sat }}\right) \\
(7)\end{array}$ & $\begin{array}{c}A_{\text {det }} \\
\left(\mathrm{m}^{2}\right) \\
(8)\end{array}$ & (9) & $\begin{array}{l}\sigma^{\prime} \\
(10)\end{array}$ & $\begin{array}{c}\text { Fluence U.L. }{ }^{\mathrm{a}, \mathrm{b}} \\
\left(\mathrm{erg} \mathrm{cm}^{-2}\right)\left(f_{\text {sat }}\right) \\
(11)\end{array}$ & $\begin{array}{c}\text { Fluence U.L. }{ }^{\mathrm{b}, \mathrm{c}} \\
\left(\mathrm{erg} \mathrm{cm}^{-2}\right)\left(f_{2.5}\right) \\
(12)\end{array}$ & $\begin{array}{c}E_{\text {cut }} \text { U.L. }{ }^{\mathrm{d}} \\
(\mathrm{GeV}) \\
(13)\end{array}$ \\
\hline $110625 \mathrm{~A}$ & Swift & 44.5 & 52.3 & 40 & 2.0 & 1.44 & 5892 & -0.15 & -0.38 & $4.9 \times 10^{-4}$ & $1.2 \times 10^{-3}$ & 3 \\
\hline $110626 \mathrm{~A}$ & Fermi & 6.4 & 9.9 & 40.4 & 1.4 & CPL & 5892 & 0.19 & 0.41 & $\ldots$ & $4.3 \times 10^{-4}$ & $\ldots$ \\
\hline $110629 \mathrm{~A}$ & Fermi & 61.7 & 70.6 & 5.1 & 1.9 & CPL & 6065 & 1.23 & 1.53 & $\ldots$ & $2.8 \times 10^{-4}$ & $\ldots$ \\
\hline 110705B & Fermi & 19.2 & 29.2 & 18.8 & 1.6 & CPL & 5892 & -0.09 & -0.28 & $\ldots$ & $9.5 \times 10^{-5}$ & $\ldots$ \\
\hline $110709 \mathrm{~A}$ & Swift & 44.7 & 46.7 & 13.5 & 2.3 & 1.24 & 6021 & 0.48 & 0.34 & $9.4 \times 10^{-5}$ & $2.3 \times 10^{-4}$ & $<2$ \\
\hline $110709 \mathrm{C}$ & Fermi & 24.1 & 32.6 & 26.7 & 2 & CPL & 5935 & -0.39 & -0.21 & & $2.2 \times 10^{-4}$ & $\ldots$ \\
\hline $110820 \mathrm{~A}$ & Swift & 256 & 256.0 & 41.6 & 4.7 & 1.92 & 5978 & 1.63 & 1.63 & $9.6 \times 10^{-3}$ & $1.6 \times 10^{-2}$ & $\ldots$ \\
\hline $110915 \mathrm{~A}$ & Swift & 78.8 & 95.7 & 39.5 & 1.9 & CPL & 5848 & 1.01 & 0.65 & $\ldots$ & $2.8 \times 10^{-3}$ & $\ldots$ \\
\hline 110919A & Fermi & 35.1 & 46.3 & 42.6 & 1.7 & CPL & 5848 & 0.04 & 0.33 & $\ldots$ & $1.3 \times 10^{-3}$ & $\ldots$ \\
\hline $110921 \mathrm{~A}$ & Swift & 48.0 & 55.6 & 7.2 & 2.1 & 1.57 & 5762 & 1.98 & 1.82 & $1.6 \times 10^{-4}$ & $3.3 \times 10^{-4}$ & 12 \\
\hline $110928 \mathrm{~B}$ & Fermi & 148.2 & 161 & 8.5 & 2 & 1.92 & 5068 & 0.26 & -0.03 & $2.5 \times 10^{-4}$ & $4.0 \times 10^{-4}$ & $\ldots$ \\
\hline $111017 \mathrm{~A}$ & Fermi & 11.1 & 17.7 & 40 & 1.4 & CPL & 5892 & -2 & -0.16 & $\ldots$ & $3.3 \times 10^{-4}$ & $\ldots$ \\
\hline $111024 \mathrm{C}$ & Fermi & 1.8 & 2.6 & 32.2 & 1.2 & CPL & 3812 & -0.99 & 0.26 & $\ldots$ & $3.7 \times 10^{-5}$ & $\ldots$ \\
\hline $111103 \mathrm{~B}$ & Swift & 167 & 167.0 & 41.6 & 3.0 & 1.41 & 5892 & 1.6 & 1.60 & $3.2 \times 10^{-3}$ & $8.1 \times 10^{-3}$ & 14 \\
\hline $111109 \mathrm{C}$ & Fermi & 9.7 & 11.9 & 32 & 1.9 & CPL & 5848 & 0.79 & 0.8 & $\ldots$ & $2.5 \times 10^{-4}$ & $\ldots$ \\
\hline $111113 \mathrm{~A}$ & $I P N$ & 0.5 & $\ldots$ & 28.4 & 1.4 & CPL & 5805 & 0.26 & $\ldots$ & $\ldots$ & $3.8 \times 10^{-5}$ & $\ldots$ \\
\hline $111208 \mathrm{~A}$ & Swift & 20 & 20.2 & 11.1 & 2.6 & 1.5 & 5112 & -0.97 & -0.97 & $5.6 \times 10^{-5}$ & $1.2 \times 10^{-4}$ & 6 \\
\hline $111215 \mathrm{~A}$ & Swift & 796 & 796.0 & 30.6 & 23.5 & 1.7 & 5848 & 0.65 & 0.65 & $2.0 \times 10^{-2}$ & $4.0 \times 10^{-2}$ & $\ldots$ \\
\hline $111228 \mathrm{~B}$ & Fermi & 2.9 & 4.2 & 23.9 & 1.4 & CPL & 5588 & -0.26 & 0.13 & & $2.9 \times 10^{-5}$ & $\cdots$ \\
\hline $120102 \mathrm{~A}$ & Swift & 38.7 & 38.7 & 44.8 & 2.4 & 1.59 & 5545 & 1.51 & 1.51 & $1.3 \times 10^{-3}$ & $2.8 \times 10^{-3}$ & $\cdots$ \\
\hline $120106 \mathrm{~A}$ & Swift & 61.6 & 61.6 & 35.4 & 2.6 & 1.53 & 5588 & -0.24 & -0.24 & $1.0 \times 10^{-3}$ & $2.4 \times 10^{-3}$ & $\ldots$ \\
\hline 120118B & Swift & 23.3 & 23.3 & 42.7 & 2.5 & 2.08 & 5502 & 0.79 & 0.79 & $1.3 \times 10^{-3}$ & $1.9 \times 10^{-3}$ & $\ldots$ \\
\hline $120118 \mathrm{C}$ & Fermi & 17.2 & 17.2 & 18.1 & 2.3 & CPL & 5458 & 1.1 & 1.1 & $\ldots$ & $2.5 \times 10^{-4}$ & $\ldots$ \\
\hline $120129 \mathrm{~A}$ & $I P N$ & 4 & 6.3 & 38.5 & 1.5 & 2.9 & 5718 & -0.07 & 0.29 & $5.0 \times 10^{-4}$ & $3.5 \times 10^{-4}$ & $\cdots$ \\
\hline $120202 \mathrm{~A}$ & Integral & 100 & 104.1 & 15.6 & 4.0 & $\ldots$ & 5718 & -0.14 & -0.23 & $\ldots$ & $7.6 \times 10^{-4}$ & $\ldots$ \\
\hline $120217 \mathrm{~A}$ & Fermi & 5.9 & 8.4 & 38.8 & 1.5 & CPL & 5458 & 0.79 & 1.03 & $\ldots$ & $5.3 \times 10^{-4}$ & $\ldots$ \\
\hline $120219 A$ & Swift & 90.5 & 90.5 & 32 & 3.4 & CPL & 5545 & -0.56 & -0.56 & $\ldots$ & $9.8 \times 10^{-4}$ & $\ldots$ \\
\hline $120222 \mathrm{~A}$ & Fermi & 1.1 & $\ldots$ & 44 & 1.4 & CPL & 5588 & 0.43 & $\ldots$ & $\ldots$ & $1.5 \times 10^{-4}$ & $\ldots$ \\
\hline $120223 \mathrm{~A}$ & Fermi & 14.3 & 16 & 37.6 & 2 & CPL & 5632 & 0.39 & 0.49 & $\ldots$ & $1.0 \times 10^{-3}$ & $\ldots$ \\
\hline $120226 \mathrm{~B}$ & Fermi & 14.6 & 18.1 & 36.8 & 1.9 & CPL & 5632 & -0.64 & -0.77 & $\ldots$ & $7.6 \times 10^{-4}$ & $\ldots$ \\
\hline $120509 \mathrm{~A}$ & Fermi & 0.7 & $\ldots$ & 14.2 & 1.3 & $\ldots$ & 5892 & -0.33 & $\ldots$ & $\cdots$ & $1.3 \times 10^{-5}$ & $\cdots$ \\
\hline $120512 \mathrm{~A}$ & Integral & 40 & 47.2 & 36.8 & 1.9 & CPL & 5892 & 0.06 & -0.29 & $\ldots$ & $1.5 \times 10^{-3}$ & $\ldots$ \\
\hline $120519 \mathrm{~A}$ & $I P N$ & 1.2 & $\ldots$ & 44.8 & 1.3 & CPL & 5935 & -2.02 & $\ldots$ & $\ldots$ & $6.9 \times 10^{-5}$ & $\ldots$ \\
\hline $120522 B$ & Fermi & 28.2 & 38.9 & 40.2 & 1.6 & 2.04 & 5892 & 0.75 & 1.16 & $8.4 \times 10^{-4}$ & $1.3 \times 10^{-3}$ & $\ldots$ \\
\hline $120604 \mathrm{~B}$ & Fermi & 12.0 & 16.9 & 33.5 & 1.6 & 1.73 & 5892 & 0.91 & 0.63 & $1.2 \times 10^{-4}$ & $2.4 \times 10^{-4}$ & $\ldots$ \\
\hline 120612B & Fermi & 63.2 & 71.4 & 21.5 & 1.8 & 1.57 & 4375 & 0.68 & 0.83 & $2.0 \times 10^{-4}$ & $4.0 \times 10^{-4}$ & $\ldots$ \\
\hline $120625 \mathrm{~A}$ & Fermi & 7.4 & 11.1 & 21.2 & 1.5 & 2.30 & 5805 & -0.27 & -0.46 & $5.8 \times 10^{-5}$ & $6.8 \times 10^{-5}$ & $\ldots$ \\
\hline $120630 \mathrm{~A}$ & Swift & 0.6 & $\ldots$ & 13.6 & 1.3 & 1.04 & 5632 & -0.78 & $\ldots$ & $3.5 \times 10^{-6}$ & $1.1 \times 10^{-5}$ & $<2$ \\
\hline $120703 \mathrm{C}$ & Fermi & 77.6 & 77.6 & 21.8 & 2.5 & 1.68 & 5675 & 0.32 & 0.32 & $2.6 \times 10^{-4}$ & $4.9 \times 10^{-4}$ & $\ldots$ \\
\hline 120727354 & Fermi & 0.90 & $\ldots$ & 5 & 1.3 & $\ldots$ & 5892 & -0.25 & $\ldots$ & $\ldots$ & $9.8 \times 10^{-6}$ & $\ldots$ \\
\hline 120819A & Swift & 71 & 71.0 & 42.1 & 2.8 & 1.49 & 5892 & 0.55 & 0.55 & $1.5 \times 10^{-3}$ & $3.6 \times 10^{-3}$ & $\ldots$ \\
\hline 120905657 & Fermi & 195.6 & 235.2 & 41.8 & 4.4 & $\ldots$ & 5935 & -2.03 & -0.66 & $\ldots$ & $4.9 \times 10^{-3}$ & $\cdots$ \\
\hline 120915474 & Fermi & 5.9 & 8.3 & 40.9 & 1.5 & $\ldots$ & 5935 & -0.08 & -0.2 & $\ldots$ & $4.2 \times 10^{-4}$ & $\ldots$ \\
\hline $121011 \mathrm{~A}$ & Swift & 75.6 & 75.6 & 19.3 & 2.2 & CPL & 5805 & -0.1 & -0.10 & $\ldots$ & $3.6 \times 10^{-4}$ & $\ldots$ \\
\hline $121012 \mathrm{~A}$ & Fermi & 0.45 & $\ldots$ & 24.7 & 1.3 & CPL & 5805 & 0.7 & $\ldots$ & $\ldots$ & $2.4 \times 10^{-5}$ & $\ldots$ \\
\hline $121025 \mathrm{~A}$ & MAXI/ISS & 20 & 30.6 & 6.9 & 1.4 & $\ldots$ & 3596 & 0.52 & 0.38 & $\cdots$ & $1.2 \times 10^{-4}$ & $\cdots$ \\
\hline $121108 \mathrm{~A}$ & Swift & 89 & 89.0 & 36.1 & 3.3 & 2.28 & 5718 & -0.19 & -0.19 & $3.1 \times 10^{-3}$ & $3.8 \times 10^{-3}$ & $\ldots$ \\
\hline 121113544 & Fermi & 95.5 & 95.5 & 34.5 & 3.1 & $\ldots$ & 5718 & 0.6 & 0.6 & $\ldots$ & $1.3 \times 10^{-3}$ & $\ldots$ \\
\hline $121123 \mathrm{~A}$ & Swift & 317 & 317.0 & 42.1 & 4.9 & CPL & 5935 & -0.25 & -0.25 & $\ldots$ & $1.2 \times 10^{-2}$ & $\ldots$ \\
\hline $121202 \mathrm{~A}$ & Swift & 20.1 & 24.1 & 27.2 & 2.1 & 1.59 & 5632 & 0.67 & 0.84 & $1.8 \times 10^{-4}$ & $3.9 \times 10^{-4}$ & 34 \\
\hline 130116415 & Fermi & 66.8 & 66.8 & 41.1 & 3.4 & $\ldots$ & 5718 & -0.24 & -0.24 & & $3.3 \times 10^{-3}$ & $\ldots$ \\
\hline $130122 \mathrm{~A}$ & Swift & 64 & 64.0 & 30.6 & 2.4 & 1.34 & 5762 & -0.23 & -0.23 & $2.3 \times 10^{-4}$ & $6.0 \times 10^{-4}$ & 7 \\
\hline
\end{tabular}

Notes. Column 1 is the GRB name corresponding to the detection date in UT (YYMMDD). Column 2 gives the satellite that detected the burst. Column 3 gives the burst duration $\Delta t_{90}$ as measured by the respective satellite. Column 4 gives the extended burst duration $\Delta t_{90}^{\prime}$. Column 5 gives the zenith angle with respect to the detector location. Column 6 gives the square root of the Fano factor. Column 7 reports the spectral index: "CPL" means that the spectrum measured by the satellite is better fitted with a cutoff power law. In case of double power law fit (Band or SBPL functions) the higher energy spectral index is reported (in italics). Column 8 gives the detector active area for that burst. Columns 9 and 10 give the statistical significance of the on-source counts over the background for standard and extended burst duration. Columns 11 and 12 give the 99\% confidence upper limits on the fluence between 1 and $100 \mathrm{GeV}$ for spectral index of Column 7 and fixed value -2.5 , respectively. Column 13 gives the cutoff upper limit, if any.

${ }^{\text {a }}$ Using the spectrum determined by satellites.

b $99 \%$ c.1.

${ }^{c}$ Assuming a differential spectral index 2.5 .

${ }^{\mathrm{d}}$ Derived from the $f_{\text {sat }}$ fluence U.L. (see the text). 


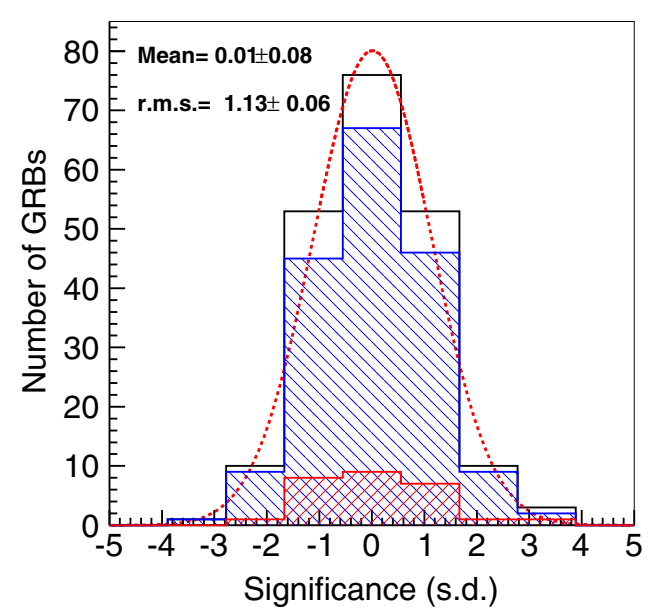

Figure 2. Distribution of the statistical significances of the 206 GRBs with respect to background fluctuations (dark solid line) compared with a free Gaussian fit (dotted line). Mean value and rms of the fit are shown. The light and dark dashed distributions refer to long and short GRBs, respectively.

(A color version of this figure is available in the online journal.)

the afterglow phase $\left(t \geqslant \Delta t_{90}\right)$ and a constant flux during the GRB prompt emission since we consider only the time-averaged behavior:

$$
\begin{array}{ll}
A(t)=A_{0} & \left(t \leqslant \Delta t_{90}\right) \\
A(t)=A_{0}\left(t / \Delta t_{90}\right)^{-3 / 2} & \left(t>\Delta t_{90}\right),
\end{array}
$$

with $A_{0}$ corresponding to the mean flux during the low-energy emission time $\Delta t_{90}$. With this assumption, two-thirds of the total emission comes after $\Delta t_{90}$. To search for such a delayed emission, a longer time interval $\Delta t_{90}^{\prime}$ has to be used. Its value is chosen in order to maximize the signal significance. Assuming Poissonian fluctuations and introducing a mean background counting rate $k$ in units of $\Delta t_{90}$, the significance is

$$
\sigma(t)=\frac{\int_{0}^{t} A(t) d t}{\sqrt{k \cdot t / \Delta t_{90}}} .
$$

The maximum of this function is at $t / \Delta t_{90}=16 / 9$. In our case, since the fluctuations are not purely Poissonian and the Fano factor $F$ depends on the integration time, we searched for a maximum significance of the modified function

$$
\sigma^{\prime}(t)=\sigma(t) / \sqrt{F(t)}
$$

with an iterative procedure, increasing for each GRB the time window by the minimum $0.5 \mathrm{~s}$ step. The Fano factor is then calculated, giving the resulting significance from Equation (6). This procedure is repeated covering the time interval from $\Delta t_{90}$ to $2 \Delta t_{90}$. The significance curve in this time window is then fitted by a second-order polynomial and the $\Delta t_{90}^{\prime}$ corresponding to its maximum is used instead of $\Delta t_{90}$ for this extended search. Since the Fano factor increases with time, $\Delta t_{90}^{\prime}$ is always shorter than the purely Poissonian value and certainly fall into the search interval. This procedure searches for a maximum in the $\left[\Delta t_{90}-2 \Delta t_{90}\right]$ range in steps of $0.5 \mathrm{~s}$, therefore the analysis has been limited to GRBs with $\Delta t_{90} \geqslant 1.5 \mathrm{~s}$, allowing a secondorder fit of function (6). Moreover, for the longer GRBs, the Fano factor is so big that the increase of $\Delta t_{90}$ does not improve the sensitivity and a clear maximum cannot be found. For these events, $\Delta t_{90}^{\prime}=\Delta t_{90}$ has been used since this is the value that

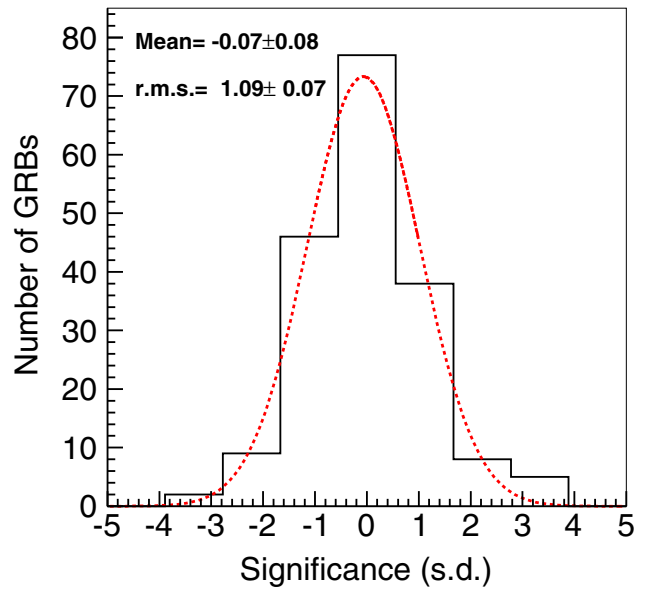

Figure 3. Distribution of the statistical significances of the $185 \mathrm{GRBs}$ with $\Delta t_{90} \geqslant 1.5 \mathrm{~s}$ with respect to background fluctuations (solid line) compared with a free Gaussian fit (dotted line) for the extended time window search (see the text). Mean value and rms of the fit are shown.

(A color version of this figure is available in the online journal.)

maximizes the signal to noise ratio for a constant signal during $\Delta t_{90}$. The $\Delta t_{90}^{\prime}$ obtained for the $185 \mathrm{GRBs}$ with $\Delta t_{90} \geqslant 1.5 \mathrm{~s}$ are listed in Tables 1 and $2\left(\Delta t_{90}^{\prime}=\Delta t_{90}\right.$ for 61 of them). The corresponding significance distribution is shown in Figure 3. No significant excess is found also in this case, the larger being $3.52 \sigma$ for GRB $080727 \mathrm{C}$ with a post-trial chance probability of $4.1 \times 10^{-2}$.

\subsection{Stacked Analysis}

Besides the coincidence analysis for each GRB, a stacked analysis has been carried out in order to search for common features of all GRBs in Time or in Phase.

In the Time analysis the counting rates for all the GRBs, in nine windows $(\Delta t=0.5,1,2,5,10,20,50,100$, and $200 \mathrm{~s})$ starting at $t_{0}$, have been added up in order to investigate a possible common duration of the high-energy emission. A positive observation at a fixed $\Delta t$ could be used as an alternative value to the observed $\Delta t_{90}$ duration and a different way to look for a possible high-energy delayed component. Since the bins are not independent, the distribution of the significances of the nine time intervals is compared with random distributions obtained for starting times different from $t_{0}$ in a time interval $\pm 12 \mathrm{hr}$ around the true GRB trigger time. Moreover, for the sample of GRBs with known redshift (with z ranging from 0.48 to 5.6), the time windows have been corrected for the cosmological dilation factor $(1+z)$. The most significant excess $(1.5 \sigma)$ is observed for the sample of 182 GRBs with no redshift at $\Delta t=0.5 \mathrm{~s}$ with a chance probability of 0.60 , while the analysis of the 24 GRBs with measured redshift led to a maximum significance of $0.7 \sigma$ in the shorter time window $(\Delta t=0.5 \mathrm{~s}$ at $z=0)$.

In the Phase analysis, only $165 \mathrm{GRBs}$ with duration $\Delta t_{90} \geqslant 5 \mathrm{~s}$ have been added up, scaling their duration to a common phase plot (i.e., 10 bins each sampling a $10 \%$ wide interval of $\Delta t_{90}$, being $0.5 \mathrm{~s}$ the minimum duration for the scaler mode data acquisition). This analysis should point out a common feature of all GRBs in case of a GeV emission correlated with the GRB duration at lower energy. Even in this case, no excess is found and the most significant bin, corresponding to the phase [0.7-0.8] of $\Delta t_{90}$, has a marginal significance of $1.78 \sigma$. 


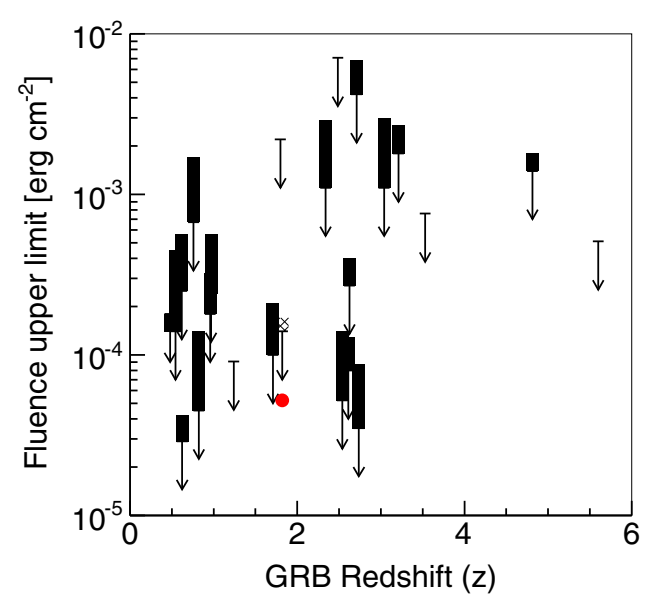

Figure 4. Fluence upper limits of GRBs in the $1-100 \mathrm{GeV}$ interval as a function of redshift. The rectangles represent the values obtained with differential spectral indexes ranging from $\alpha=-2.5$ to the satellite measurement $\alpha_{\text {sat }}$. The five arrows give the upper limits for the former case only, these GRBs being best fitted at lower energies with a cutoff power law spectrum. The dot shows the fluence extrapolated in the $1-100 \mathrm{GeV}$ range from the Fermi-LAT observations of GRB 090902B; only for this GRB, the GeV spectral index has been used and the dashed area has been obtained applying an energy cutoff running from 30 to $100 \mathrm{GeV}$.

(A color version of this figure is available in the online journal.)

\section{FLUENCE AND $E_{\text {CUT }}$ UPPER LIMITS}

The fluence upper limits can be derived in the [1-100] GeV range from our experimental data and making some assumptions on the GRB primary spectrum. For this calculation, we used the maximum number of counts at $99 \%$ confidence level (c.l.) following Equation (6) of Helene (1983). The interaction of the GRB photons with the EBL results in $e$-pair production, which originates a spectral cutoff. This effect depends on the GRB redshift, with a lower cutoff energy for more distant GRBs. For this reason, the most meaningful upper limits are obtained for the sample of 24 GRBs with known redshift (see table 1), while for the others (Table 2) a value of $z=2$ and $z=0.6$ has been adopted for long and short GRBs, respectively, according to their measured distributions (Jakobsson et al. 2006; Berger et al. 2005; Berger 2014). For the differential spectral indexes, we used two extrapolations to estimate the expected high-energy fluence for each GRB: (a) the spectral index $\alpha_{\text {sat }}$ measured by satellite detectors in the $\mathrm{keV}-\mathrm{MeV}$ energy range (corresponding to the $f_{\text {sat }}$ values in Tables 1 and 2), and (b) the conservative value $\alpha=-2.5$ ( $f_{2.5}$ values in Tables 1 and 2). For case (a), when the Band or SBPL spectral features have been identified, the higher energy spectral index (i.e., above the peak in the $\mathrm{keV}-\mathrm{MeV}$ region) has been used. These assumptions represent respectively the most and less favorable spectral index hypotheses. The absorption effect due to the EBL is taken into account using the model described in Kneiske et al. (2004) and applying an exponential cutoff to the spectrum according to the redshift. Figure 4 shows the 99\% c.l. upper limits as a function of $\mathrm{z}$ for the GRBs with known redshift. For five of them, whose spectrum is best fitted by a CPL, only the upper limits for case (b) are given.

For GRB 090902B (which was the GRB in the ARGO-YBJ field of view with the highest energy photon detected), the fluence extrapolated from Fermi-LAT observations in the same energy range is shown. Only for this GRB, the GeV spectral index measured by Fermi-LAT has been used and the dashed area in Figure 4 has been obtained applying an energy cutoff to the GRB spectrum running from $30 \mathrm{GeV}$ (about the maximum

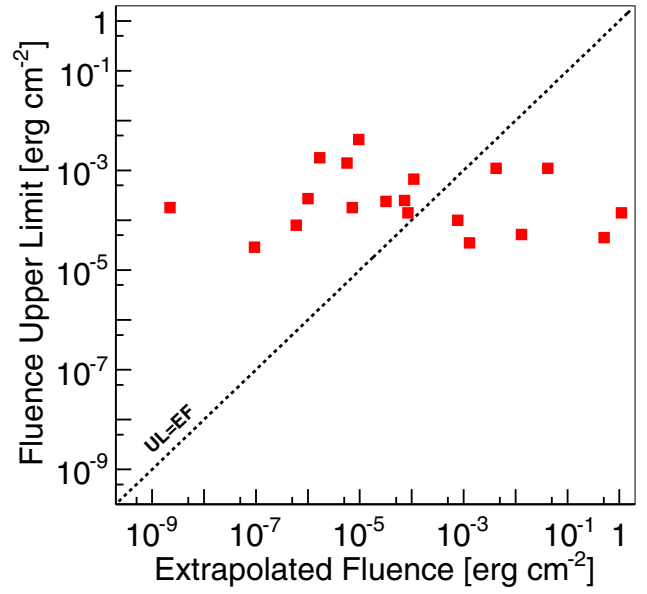

Figure 5. ARGO-YBJ upper limits (in the $1-100 \mathrm{GeV}$ interval) vs. fluence extrapolation for GRBs with measured redshift and low-energy power-law spectral index.

(A color version of this figure is available in the online journal.)

energy measured by Fermi-LAT) to $100 \mathrm{GeV}$. According to our calculation, in the case of a spectrum extending up to $100 \mathrm{GeV}$, the extrapolated GRB fluence is just a factor of 2.7 lower than our expected sensitivity. Due to the peculiar $\mathrm{GeV}$ emission of this GRB, the search has also been done in different time windows, in particular in coincidence with the extended Fermi-LAT emission [0-90 s], the maximum density of events with energy $>1 \mathrm{GeV}$ [6-26 s], and the time of the 33.4 $\mathrm{GeV}$ photon $[82-83 \mathrm{~s}]$. The resulting significances are $-0.03 \sigma$, $1.00 \sigma$, and $-0.52 \sigma$, respectively.

A comparison between the expected fluence, obtained extrapolating the $\mathrm{keV}-\mathrm{MeV}$ spectra measured by satellites and including the EBL absorption, and the fluence upper limit determined with the ARGO-YBJ scaler data has been done for the 19 GRBs with measured redshift and energy spectrum best fitted by a simple power law, excluding the five events that present a CPL spectrum. The result is shown in Figure 5. The seven points on the right side of the line upper limit (UL) = expected fluence (EF) (i.e., in the region where the upper limits are lower than the extrapolated fluences) indicate that since the corresponding GRBs were not detected, the chosen extrapolation is not feasible up to our range [1-100 GeV] or a cutoff should be present in the high-energy tail of the spectrum. Therefore, assuming the spectral index measured at low energies, the maximum cutoff energy has been estimated as follows. The extrapolated fluence is calculated together with the fluence upper limit as a function of the cutoff energy $E_{\text {cut }}$. If the two curves cross in the [2-100 GeV] interval, the intersection gives the upper limit to the cutoff energy. This is what happens to four GRBs (GRB 050802, GRB 081028A, GRB 090809A, and GRB 110128A) for which the knowledge of the redshift allows the estimation of the extragalactic absorption and hence a more accurate fluence upper limit and cutoff energy determination. For three GRBs (GRB 071112C, GRB 090424, and GRB 130113B), the estimated $E_{\text {cut }}$ upper limit is below $2 \mathrm{GeV}$ : we can conclude that in these cases, the low-energy spectrum cannot be extended to the $\mathrm{GeV}$ region and some additional features occur in the $\mathrm{keV}-\mathrm{MeV}$ range. The values obtained for $E_{\text {cut }}$ are reported in the last column of Table 1 and shown in Figure 6 (triangles) as a function of the spectral index. The same calculation can be made for the GRBs with unknown redshift assuming for the EBL absorption $z=2$ and $z=0.6$ for long and short GRBs, respectively, and the 


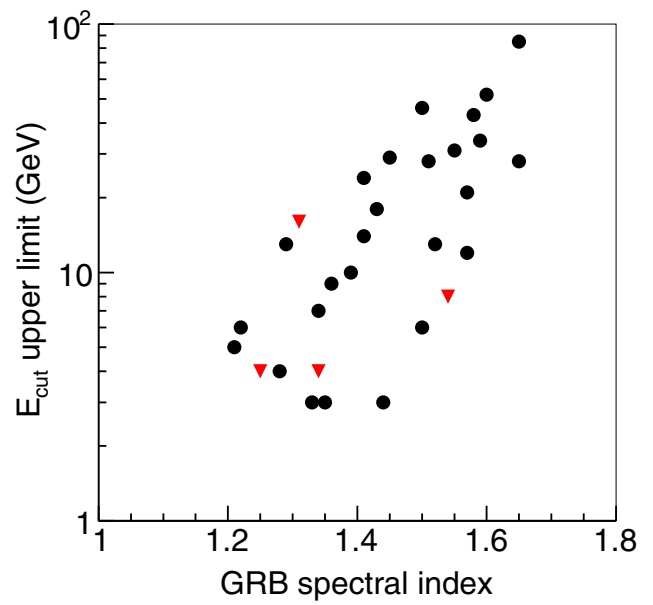

Figure 6. Cutoff energy upper limits as a function of the spectral index obtained by extrapolating the measured $\mathrm{keV}$ spectra. The values represented by the triangles are obtained taking into account extragalactic absorption at the known GRB redshift. For the other GRBs (dots), $z=2$ and $z=0.6$ are assumed for long and short ones, respectively.

(A color version of this figure is available in the online journal.)

resulting $E_{\text {cut }}$ values are given in the last column of Table 2 and shown in Figure 6 (dots). More realistic models for the spectrum shape and/or different hypotheses on the photon spectral index in the $\mathrm{GeV}$ region can be considered. Since all seven GRBs falling on the right side of the $\mathrm{UL}=\mathrm{EF}$ line in Figure 5 are long, we first assumed a Band spectrum with an $E_{\text {peak }}$ value of $160 \mathrm{keV}$ and a spectral index $\beta=-2.34$, corresponding to the mean peak energy and high-energy slope for this class of GRBs (Nava et al. 2011). With this model, all seven GRBs result under threshold (i.e., the extrapolated fluence is lower than our upper limit).

Another possibility is to suppose a fixed ratio between the $\mathrm{GeV}$ and $\mathrm{keV}-\mathrm{MeV}$ fluences. The simultaneous observation of GRBs in these energy bands has been performed in the past by EGRET and BATSE on board the Compton Gamma Ray Observatory satellite and more recently by Fermi-LAT and Fermi-GBM for a handful of events. As pointed out by Dermer et al. (2010), for long GRBs, this ratio is close to 0.1 when the energy ranges considered to determine the fluence are $100 \mathrm{MeV}-10 \mathrm{GeV}$ and $20 \mathrm{keV}-2 \mathrm{MeV}$. We used a value of -2 for the GeV spectral index, consistent with both EGRET and FermiLAT mean values. This high-energy component represents a strong deviation with respect to the Band spectrum, increasing significantly the expected $\mathrm{GeV}$ fluence even if to a smaller extent than extrapolating the $\mathrm{keV}-\mathrm{MeV}$ spectra. Also under these hypotheses, all seven long GRBs fall on the left side of the $\mathrm{UL}=\mathrm{EF}$ line in Figure 5.

\section{DISCUSSION AND CONCLUSIONS}

The detection of high-energy photons by the Fermi-LAT instrument clearly demonstrates that at least a small fraction of GRBs emit in the GeV range. The detected photons experience two main processes: generation at the source and propagation through the intergalactic medium. Several models have been proposed to explain the production of high-energy photons in GRBs, but according to the standard fireball shock model, they are essentially caused by internal or external shocks. Once produced, a fraction of these photons are converted into electron-positron pairs due to the interaction with low-energy photons, mainly of the infrared-optical-ultraviolet cosmic back- ground (EBL). This mechanism limits the photon mean free path and thus the visible horizon, which decreases with the energy up to $\sim 10^{15} \mathrm{eV}$, where the interaction with the cosmic microwave background radiation makes it smaller than the Galactic radius. The signal reaching the Earth is the final result of all these production and propagation mechanisms, bringing valuable information on all of them but at the same time making them difficult to separate. Features like the maximum energy as a function of the redshift, the photon index, and other temporal and spectral characteristics, if seen with sufficient statistics, could discriminate between different mechanisms and shed light in this still largely unknown field. For these reasons, the study of GRBs would greatly benefit from the contribution of ground-based detectors to the direct satellite measurements.

In this paper, a search for GRBs in coincidence with satellite detections has been carried out using the complete ARGO-YBJ data set. During about eight years, a total of 206 GRBs has been analyzed, producing the largest GRB sample ever studied using the scaler mode technique. In the search for $\mathrm{GeV} \gamma$-rays in coincidence with the GRB satellite detections, no evidence of emission was found for any event both for the whole sample and for separate analyses of the two populations of long and short GRBs. For GRBs with duration $\geqslant 1.5 \mathrm{~s}$, the search for a signal in a time window extended with respect to the lowenergy one has been carried out with similar results. The stacked search, both in time and phase, has shown no deviation from the statistical expectations. The subset of 24 GRBs with known redshift has been carefully analyzed in terms of fluence and cutoff upper limits. For GRB 090902B, the fluence upper limit using the GeV spectral index is very close to the Fermi-LAT measurement (a factor of 2.7 higher), supposing a high-energy emission extending from the observed $30 \mathrm{GeV}$ up to $100 \mathrm{GeV}$. This GRB was certainly our best candidate for a detection; however, an area 7.2 times larger would have been necessary. For the other GRBs with known redshift, fluence upper limits as low as $2.9 \times 10^{-5} \mathrm{erg} \mathrm{cm}^{-2}$ in the $1-100 \mathrm{GeV}$ energy range have been set, assuming a high-energy spectral index equal to that measured by satellites. Under this hypothesis, an upper limit to the cutoff energy has also been determined for seven GRBs, otherwise an average Band spectrum or a fixed ratio between the high and low-energy fluences must be assumed.

The expected rate of GRBs that could be observed by the ARGO-YBJ experiment, based on the Swift satellite detections, was between 0.1 and $0.5 \mathrm{yr}^{-1}$ (Aielli et al. 2008) and it should have doubled with the later launch of the Fermi satellite. The value of $0.3 \mathrm{yr}^{-1}$ obtained for our $90 \%$ c.l. upper limit is close to our lower expectation partially because the predicted Fermi detection rate was overestimated and partially because the LATdetected GRBs have a spectrum softer than presumed.

In the next future, three huge ground-based detectors could continue this search with improved sensitivity. HAWC, a water Cherenkov detector with a surface of $22,000 \mathrm{~m}^{2}$ is under construction in Mexico at an altitude of $4100 \mathrm{~m}$ a.s.1.. Its expected detection rate is $1.55 \mathrm{yr}^{-1}$ for short GRBs and $0.25 \mathrm{yr}^{-1}$ for long GRBs, mainly using the shower mode technique in the range 50-500 GeV (Taboada \& Gilmore 2014). CTA will observe the night sky detecting the atmospheric Cherenkov light. Its huge telescopes for the detection of low-energy $\gamma$-rays have been designed also for fast slewing, allowing a repointing time of $\lesssim 100 \mathrm{~s}$. Apart from a very lucky serendipitous observation, the CTA search is limited to long GRBs after the very prompt phase, with an expected detection rate ranging from 0.6 to $2 \mathrm{yr}^{-1}$ according to baseline or optimistic assumptions and with 
a strong dependence on the energy threshold (more than on the pointing delay) (Gilmore et al. 2013). GRB detection from ground via the water Cherenkov technique will also be possible with the proposed LHAASO experiment (Cui et al. 2014), whose detection rate has not yet been estimated. Thirty years after the first proposal by Morello et al. (1984), the first solid detection of a GRB from the ground seems at hand.

This work was supported in China by NSFC (grant No. 10120130794), the Chinese Ministry of Science and Technology, the Chinese Academy of Sciences, the Key Laboratory of Particle Astrophysics, CAS; and in Italy by the Istituto Nazionale di Fisica Nucleare (INFN). We also acknowledge the essential support of W. Y. Chen, G. Yang, X. F. Yuan, C. Y. Zhao, R. Assiro, B. Biondo, S. Bricola, F. Budano, A. Corvaglia, B. D’Aquino, R. Esposito, A. Innocente, A. Mangano, E. Pastori, C. Pinto, E. Reali, F. Taurino, and A. Zerbini in the installation, debugging, and maintenance of the detector.

\section{REFERENCES}

Abdo, A. A., Ackermann, M., Ajello, M., et al. 2009a, ApJL, 706, L138 Abdo, A. A., Ackermann, M., Arimoto, M., et al. 2009b, Sci, 323, 1688 Ackermann, M., Ajello, M., Asano, K., et al. 2011, ApJ, 729, 114 Ackermann, M., Ajello, M., Asano, K., et al. 2013, ApJS, 209, 11 Ackermann, M., Ajello, M., Asano, K., et al. 2014, Sci, 343, 42 Ackermann, M., Asano, K., Atwood, W. B., et al. 2010, ApJ, 716, 1178 Aielli, G., Assiro, R., Bacci, C., et al. 2006, NucIM, A562, 92 Aielli, G., Bacci, C., Barone, F., et al. 2008, APh, 30, 85 Aielli, G., Bacci, C., Barone, F., et al. 2009a, ApJ, 699, 1281 Aielli, G., Bacci, C., Bartoli, B., et al. 2009b, APh, 32, 47 Aielli, G., Bacci, C., Bartoli, B., et al. 2009c, PhRvD, 80, 092004 Aielli, G., Bacci, C., Bartoli, B., et al. 2010, ApJL, 714, L208 Aielli, G., Bacci, C., Bartoli, B., et al. 2011, ApJ, 729, 113 Aielli, G., Bacci, C., Bartoli, B., et al. 2012, NucIM, A661, S50 Atwood, W. B., Baldini, L., Bregeon, J., et al. 2013, ApJ, 774, 76 Band, D., Matteson, J., Ford, L., et al. 1993, ApJ, 413, 281 Bartoli, B., Bernardini, P., Bi, X. J., et al. 2011, ApJ, 734, 110 Bartoli, B., Bernardini, P., Bi, X. J., et al. 2012a, ApJL, 745, L22 Bartoli, B., Bernardini, P., Bi, X. J., et al. 2012b, ApJ, 758, 2 Bartoli, B., Bernardini, P., Bi, X. J., et al. 2012c, ApJ, 760, 110 Bartoli, B., Bernardini, P., Bi, X. J., et al. 2012d, PhRvD, 85, 022002 Bartoli, B., Bernardini, P., Bi, X. J., et al. 2012e, PhRvD, 85, 092005 Bartoli, B., Bernardini, P., Bi, X. J., et al. 2013a, ApJ, 767, 99 Bartoli, B., Bernardini, P., Bi, X. J., et al. 2013b, ApJ, 779, 27 Bartoli, B., Bernardini, P., Bi, X. J., et al. 2013c, PhRvD, 88, 082001 Bartoli, B., Bernardini, P., Bi, X. J., et al. 2014, Radiation Measurements, 68 , 42
Becherini, Y., Punch, M., \& H. E. S. S. Collaboration, 2012, in AIP Conf. Proc 1505, High Energy Gamma-ray Astronomy: 5th Heidelberg International Symp. on High Energy Gamma-ray Astronomy (Gamma 2012), ed. F. A. Aharonian, W. Hofmann, \& F. M. Rieger (Melville, NY: AIP), 741

Berger, E. 2014, ARA\&A, 52, 43

Berger, E., Kulkarni, S. R., Fox, D. B., et al. 2005, ApJ, 634, 501

Bernlöhr, K., Barnacka, A., Becherini, Y., et al. 2013, APh, 43, 171

Campana, S., Mangano, V., Blustin, A. J., et al. 2006, Natur, 442, 1008

Costa, E., Frontera, F., Heise, J., et al. 1997, Natur, 387, 783

Cui, S., Liu, Ye, Liu, Yujuan, \& Ma, X. 2014, APh, 54, 86

Dermer, C. D., \& Fermi Collaboration, 2010, in AIP Conf. Proc. 1279, Deciphering the Ancient Universe with Gamma-ray Bursts, ed. N. Kawai \& S. Nagataki (Melville, NY: AIP), 191

Fan, Y. Z., Piran, T., Narayan, R., \& Wei, D-M. 2008, MNRAS, 384, 1483 Fano, U. 1947, PhRv, 72, 26

Finke, J. D., Dermer, C. D., \& Böttcher, M. 2008, in AIP Conf. Proc. 1000, Gamma-ray Bursts 2007, ed. M. Galassi, D. Palmer, \& E. Fenimore (Melville, NY: AIP), 385

Gal-Yam, A., Moon, D.-S., Fox, D. B., et al. 2004, ApJL, 609, L59

Ghirlanda, G., Ghisellini, G., \& Nava, L. 2010, A\&A, 510, L7

Ghisellini, G., Ghirlanda, G., Nava, L., \& Celotti, A. 2010, MNRAS, 403, 926

Gilmore, R C., Bouvier, A., Connaughton, V., et al. 2013, ExA, 35, 413

Giroletti, E., Bolognino, I., Cattaneo, C., et al. 2011, in Proc. 32nd ICRC (Beijing), 1, 18

Gruber, D., Goldstein, A., Weller von Ahlefeld, V., et al. 2014, ApJS, 211, 12

Guetta, D., \& Granot, J. 2003, ApJ, 585, 885

Helene, O. 1983, NIMPR, 212, 319

Jakobsson, P., Levan, A., Fynbo, J. P. U., et al. 2006, A\&A, 447, 897

Kanbach, G., Bertsch, D. L., Favale, A., et al. 1988, SSRv, 49, 69

Kaneko, Y., Preece, R. D., Briggs, M. S., et al. 2006, ApJS, 166, 298

Kneiske, T. M., Bretz, T., Mannheim, K., \& Hartmann, D. H. 2004, A\&A, 413, 807

Kocevski, D., Vianello, G., Chiang, J., et al. 2013, GCN, 14532

Kumar, P., \& Barniol Duran, R. 2010, MNRAS, 409, 226

Li, T., \& Ma, Y. 1983, ApJ, 272, 317

Longo, F., Moretti, E., Nava, L., et al. 2012, A\&A, 547, A95

Meegan, C., Lichti, G., Bhat, P. N., et al. 2009, ApJ, 702, 791

Morello, C., Periale, L., \& Navarra, G. 1984, NCimC, 7, 682

Nava, L., Ghirlanda, G., Ghisellini, G., \& Celotti, A. 2011, A\&A, 530, A21

Rosswog, S., Ramirez-Ruiz, E., \& Davies, M. B. 2003, MNRAS, 345, 1077

Ruffert, M., \& Janka, H.-Th. 1999, A\&A, 344, 573

Stanek, K. Z., Matheson, T., Garnavich, P. M., et al. 2003, ApJL, 591, L17

Taboada, I., \& Gilmore, R. C. 2014, NucIM, A742, 276

Tanvir, N. R., Levan, A. J., Fruchter, A. S., et al. 2013, Natur, 500, 547

Vernetto, S. 2000, APh, 13, 75

Vianello, G., Desiante, R., \& Longo, F. 2013, GCN, 15357

von Kienlin, A., Meegan, C. A., Paciesas, W. S., et al. 2014, ApJS, 211, 13

Wang, X. Y., Cheng, K. S., Dai, Z. G., \& Lu, T. 2005, A\&A., 439, 957

Weiler, K. W., Panagia, N., \& Montes, M. J. 2001, ApJ, 562, 670

Zhou, X. M., Ye, N., Zhu, F. R., et al. 2011, in Proc. 32nd ICRC (Beijing), 11, 287 